\title{
Genetic-Algorithm-Assisted Sliding Mode Control for Networked State-Saturated Systems over Hidden Markov Fading Channels
}

\author{
Jun Song, Member, IEEE, Zidong Wang, Fellow, IEEE, Yugang Niu, Hongli Dong, Senior Member, IEEE
}

\begin{abstract}
The sliding mode control (SMC) problem is studied in this paper for state-saturated systems over a class of timevarying fading channels. The underlying fading channels, whose channel fading amplitudes (characterized by the expectation and variance) are allowed to be different, are modeled as a finitestate Markov process. A key feature of the problem addressed is to use a hidden Markov mode detector to estimate the actual network mode. The novel model of hidden Markov fading channels is shown to be more general yet practical than the existing fading channel models. Based on a linear sliding surface, a switchingtype SMC law is dedicatedly constructed by just using the estimated network mode. By exploiting the concept of stochastic Lyapunov stability and the approach of hidden Markov models, sufficient conditions are obtained for the resultant SMC systems that ensure both the mean-square stability and the reachability with a sliding region. With the aid of the Hadamard product, a binary genetic algorithm (GA) is developed to solve the proposed SMC design problem subject to some nonconvex constraints induced by the state saturations and the fading channels, where the proposed GA is based on the objective function for optimal reachability. Finally, a numerical example is employed to verify the proposed GA-assisted SMC scheme over the hidden Markov fading channels.
\end{abstract}

Index Terms - Sliding Mode Control; Hidden Markov Model; State Saturation; Finite-State Fading Channels; Genetic Algorithm.

\section{INTRODUCTION}

Sliding mode control (SMC) has been recognized as one of the most effective robust control approaches to tackling nonlinearities and external disturbances. Various SMC techniques have been successfully applied to a wide range of

This work was supported in part by the National Natural Science Foundation of China under Grants 61903143, 61933007, 61873058, 61873148, 61673174 and 61773162, the Research Fund for the Taishan Scholar Project of Shandong Province of China, the Shanghai Sailing Program of China under Grant 19YF1412100, the 111 Project of China under Grant B17017, the Royal Society of the U.K., and the Alexander von Humboldt Foundation of Germany. (Corresponding author: Zidong Wang.)

J. Song and Y. Niu are with the Key Laboratory of Advanced Control and Optimization for Chemical Process (East China University of Science and Technology), Ministry of Education, Shanghai 200237, China (Emails: sjky5211@163. com; acniuyg@ecust.edu.cn).

Z. Wang is with the College of Electrical Engineering and Automation, Shandong University of Science and Technology, Qingdao 266590, China $\mathrm{He}$ is also with the Department of Computer Science, Brunel University London, Uxbridge, Middlesex, UB8 3PH, United Kingdom. (Email: Zidong.Wang@brunel.ac.uk).

H. Dong is with the Institute of Complex Systems and Advanced Control, Northeast Petroleum University, Daqing 163318, China, and are also with the Heilongjiang Provincial Key Laboratory of Networking and Intelligent Control, Northeast Petroleum University, Daqing 163318, China. (Email: shiningdhlevip.126. com). engineering systems such as power electronics, mechanical systems, and chemical processes. Over the past three decades, a large number of research results have been developed for SMC problems of continuous-time systems [1], [2], [17] and discrete-time systems [13], [14], [23].

As a typical kind of nonlinear systems, state-saturated systems possess the distinctive characteristics that the states are constrained into a prescribed bounded area quantified by saturation level. The state saturation phenomenon occurs frequently in engineering practice owing to physical limitations of the devices and/or protection equipment's [28], [29], [43]. By introducing a polytopic representation of saturation nonlinearities, some representative results have been acquired in [8], [16] on various dynamics analysis issues (e.g. stability, robustness, filtering and control) for state-saturated systems. Nonetheless, the SMC problem for state-saturated systems remains an open yet interesting research problems.

For decades, networked control systems (NCSs) have been gaining considerable research interest in response to the rapid spread of communication network. The main feature of NCSs is that the data transmission between different system components is executed via communication networks, and this renders the advantages of low cost, simple installation and high reliability [18], [30], [31], [42], [44], [46]. Unfortunately, the limited bandwidth of the network channels may give rise to various networked-induced complexities that could cause performance degradation or even instability of the NCSs. Till now, the SMC issues have been under intensive investigation for NCSs subject to various network-induced phenomena including packet dropout [23], [32], signal quantization [15], [21], randomly occurring incomplete information [20], [36], and communication protocols [33], [35].

As an inevitable network-induced phenomenon in wireless communication networks, the channel fading problem has stirred much research attention, see e.g. [4], [11], [27] and the references therein. Reflection, refraction and diffraction may physically cause the fluctuation of wireless signals that leads to the channel fading. In general, a signal can be delivered through analog or digital pathways. In analog fading channel, the signal fluctuation can be characterized by a multiplicative noise model and, in digital fading channel, the packet-loss rate is usually dependent on the channel power gain, power level and packet length. So far, the control/filtering issues have been discussed in [9], [11], [39] for analog fading channel and in [6], [26] for digital fading channel. Nevertheless, the SMC problem subject to fading channels has not received adequately 
research attention yet, which is still an ongoing research issue.

In the real world, the channel fading phenomenon occurs typically in a time-varying fashion. In other words, the amplitude and/or phase of the transmitted signal may experience fadings caused by the changing communication environment. It has been shown that the wireless flat-fading channels can be well described by the model of finite-state Markov fading channels (FSMFCs), where the stochastic time-varying feature is captured by a finite-state Markov process [27]. Up to now, the filtering and stabilization problems over FSMFCs have been addressed in some pioneering work, see e.g. [26], [38], [40]. From the viewpoint of practical applications, a key problem in employing the FSMFCs model is how to estimate the actual network mode based on the measured network information. In [38], an estimation/observation model has been proposed for the actual network mode that covers three special cases, namely, the current network mode observation, one-step delayed network mode observation and no observation/estimation of network mode. Motivated by the work in [38], a seemingly interesting question is whether it is possible to develop a more general yet simple estimation model to not only cover the above three special cases but also observe the multi-step delayed network mode, and one of the motivations of this paper is therefore to answer such a question.

According to the above discussion, it makes both theoretical and practical sense to study the SMC design for networked state-saturated systems over FSMFCs. Very recently, the mode detector approach has been put forward to describe the asynchronous phenomena between the Markovian jump system modes and the controller/filtering modes [5], [7], [24], [37]. Inspired by such an approach, in this paper, we focus our attention on the design task of the SMC scheme for statesaturated systems over hidden Markov fading channels (HMFCs), in which the jumping network mode of the FSMFCs is estimated/observed by a mode detector via a hidden Markov model (HMM). It is worth pointing out that the addressed task is not easily accomplishable due to the following three essential obstacles: 1) how to estimate the actual network mode based on the hidden Markov detection mode? 2) how to construct the sliding surface and the desired SMC law by just using the estimated network mode information? 3) how to analyze the stability and the reachability under the state saturation and the HMFCs? The aim of this paper is to provide satisfactory responses to these three questions through launching a systematic investigation.

This paper endeavors to design the sliding mode controller for a class of state-saturated NCSs over the HMFCs, where the actual network mode over the fading channels is estimated by a mode detector via a HMM. The novelties of this paper lie in the following four aspects:

1) Based on a novel fading channels model of HMFCs, the SMC problem is, for the first time, investigated for the networked state-saturated systems over the fading channels.

2) Based on a linear sliding surface, a switching SMC strategy is proposed by using the estimated network mode only.
3) By exploiting the polytopic representation of the state saturation and the HMM approach, both the meansquare stability and the reachability are studied for the SMC system.

4) With the aid of the Hadamard product, a genetic algorithm (GA) is formulated to solve the proposed SMC design problem subject to some nonconvex constraint conditions resulting from the state saturation and the fading channels.

Notations. $\mathbb{R}^{n}$ represents the set of $n$-dimensional real numbers. $\mathbb{N}_{0}$ represents the set including zero and all positive integers, i.e., $\{0,1,2, \ldots\}$. The expectation operator is denoted as $\mathbf{E}\{\cdot\} \cdot \operatorname{Pr}\{A \mid B\}$ represents the conditional probability of the event $A$ under the the event $B$. The block diagonal matrix is denoted by "diag $\{\cdot\}$ ". For the real matrices $A=\left[a_{i j}\right]_{m \times n}$ and $B=\left[b_{i j}\right]_{m \times n}$, the Hadamard product $A \circ B$ is defined as $A \circ B \triangleq\left[a_{i j} b_{i j}\right]_{m \times n} \cdot \operatorname{sgn}(\cdot)$ denotes the standard sign function.

\section{Problem Formulation}

\section{A. State-saturated systems}

This paper is concerned with the problem of sliding mode stabilization over HMFCs for the following state-saturated systems:

$x(k+1)=\sigma((A+\Delta A(k)) x(k)+B(u(k)+\phi(x(k), k)))$,

where $x(k) \in \mathbb{R}^{n}$ is the state vector, $u(k) \in \mathbb{R}^{m}$ is the actuator input vector, and $\phi(x(k), k) \in \mathbb{R}^{m}$ is the matched input disturbance satisfying $\|\phi(x(k), k)\| \leq \varphi\|x(k)\|$ with a known scalar $\varphi \geq 0$. The norm-bounded parameter uncertainty $\Delta A(k)$ satisfies $\Delta A(k)=M F(k) H$ with $F^{\mathrm{T}}(k) F(k) \leq I$. Here, $A, B, M$ and $H$ are known constant matrices, and the input matrix $B$ satisfies $\operatorname{rank}(B)=m$.

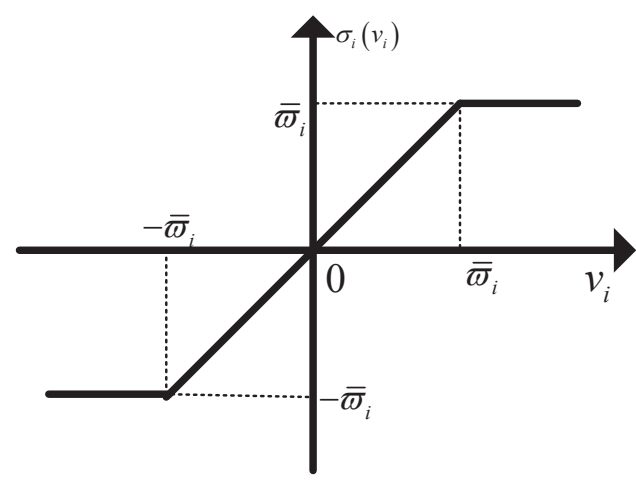

Fig. 1. The saturation function $\sigma_{i}\left(v_{i}\right)$

The state-saturated function $\sigma(\cdot): \mathbb{R}^{n} \rightarrow \mathbb{R}^{n}$ in (1) is defined as follows:

$$
\sigma(v) \triangleq\left[\begin{array}{llll}
\sigma_{1}\left(v_{1}\right) & \sigma_{2}\left(v_{2}\right) & \cdots & \sigma_{n}\left(v_{n}\right)
\end{array}\right]^{\mathrm{T}}, \forall v \in \mathbb{R}^{n},
$$

where the scalar-valued saturation function $\sigma_{i}\left(v_{i}\right)$ (shown in Fig. 1) is defined by

$$
\sigma_{i}\left(v_{i}\right) \triangleq \operatorname{sgn}\left(v_{i}\right) \min \left\{\bar{\varpi}_{i},\left|v_{i}\right|\right\},
$$


with $v_{i}$ representing the $i$ th element of the vector $v$ and $\bar{\varpi}_{i}$ standing for the saturation level. To help with the analysis later, we denote

$$
\Lambda \triangleq \operatorname{diag}\left\{\bar{\varpi}_{1}, \bar{\varpi}_{2}, \ldots, \bar{\varpi}_{n}\right\} .
$$

\section{B. Hidden Markov fading channels}

In this paper, the unreliable network consists of a set of FSMFCs. Let the network mode $\varsigma(k) \in \Theta \triangleq\{1,2, \ldots, N\}$ obey a discrete-time Markov process with the following transition probabilities:

$$
\pi_{i j} \triangleq \operatorname{Pr}\{\varsigma(k+1)=j \mid \varsigma(k)=i\}, k \in \mathbb{N}_{0}, \forall i, j \in \Theta
$$

where $\pi_{i j} \in[0,1]$ and $\sum_{j=1}^{N} \pi_{i j}=1$. The transition probability matrix is defined as $\Pi \triangleq\left[\pi_{i j}\right]_{i, j \in \Theta}$. In practical application, the network modes $\varsigma(k)$ correspond to different channel fading amplitudes or different configurations of the overall physical environment (e.g., positions of mobile agents) [26], [27], [38].

Now, at the network mode $\varsigma(k)$, the network input-output behavior can be characterized by

$$
u(k)=\vartheta_{\varsigma(k)}(k) v(k),
$$

where the channel fading phenomenon is reflected in $\vartheta_{\varsigma(k)}(k) \in \mathbb{R}^{m \times m}$ that has the diagonal structure

$$
\vartheta_{\varsigma(k)}(k)=\operatorname{diag}\left\{\vartheta_{1, \varsigma(k)}(k), \vartheta_{2, \varsigma(k)}(k), \ldots, \vartheta_{m, \varsigma(k)}(k)\right\}
$$

and, for any $b=1,2, \ldots, m, \vartheta_{b, \varsigma(k)}(k)$ is scalar-valued random process with

$$
\begin{aligned}
& \mu_{b, \varsigma(k)} \triangleq \mathbf{E}\left\{\vartheta_{b, \varsigma(k)}(k)\right\}, \\
& \xi_{b l, \varsigma(k)} \triangleq \mathbf{E}\left\{\left(\vartheta_{b, \varsigma(k)}-\mu_{b, \varsigma(k)}\right)\left(\vartheta_{l, \varsigma(k)}-\mu_{l, \varsigma(k)}\right)\right\}
\end{aligned}
$$

which satisfy $\mu_{b, \varsigma(k)}>0, \xi_{b b, \varsigma(k)}>0$, and $\xi_{b l, \varsigma(k)}=\xi_{l b, \varsigma(k)}$ for all $\varsigma(k) \in \Theta$ and $b, l=1,2, \ldots, m$.

We further write

$$
\begin{aligned}
& \Gamma_{\varsigma(k)} \triangleq \operatorname{diag}\left\{\mu_{1, \varsigma(k)}, \mu_{2, \varsigma(k)}, \ldots, \mu_{m, \varsigma(k)}\right\}, \\
& \Phi_{\varsigma(k)} \triangleq\left[\xi_{b l, \varsigma(k)}\right]_{b, l=1,2, \ldots, m}, \\
& \Psi_{\varsigma(k)} \triangleq \operatorname{diag}\left\{\xi_{11, \varsigma(k)}, \xi_{22, \varsigma(k)}, \ldots, \xi_{m m, \varsigma(k)}\right\} .
\end{aligned}
$$

It is easily seen that $\Phi_{\varsigma(k)}$ is positive semidefinite.

Remark 1: It is worth mentioning that the model (6) with the Markov process (5) can also be employed to describe the jumping actuator failures [25], the quantizer with the Markovian jump quantization density [22] and the Markovian packet losses [32]. Specifically,

- for the jumping actuator failures [25], $\vartheta_{b, \varsigma(k)}(k)=0$, $0<\vartheta_{b, \varsigma(k)}(k)<1$, and $\vartheta_{b, \varsigma(k)}(k)=1$ correspond to the complete failure, partial failure, and failure-free cases of the $r$ th actuator, respectively;

- for the quantizer with the Markovian jump quantization density [22], the model (6) is specified as

$$
u(k)=\left(I_{m}+\boldsymbol{\Delta}_{\varsigma(k)}(k)\right) v(k),
$$

where $\boldsymbol{\Delta}_{\varsigma(k)}(k) \triangleq \operatorname{diag}\left\{\Delta_{1, \varsigma(k)}(k), \ldots, \Delta_{m, \varsigma(k)}(k)\right\}$, $\Delta_{l, \varsigma(k)}(k) \in\left[-\zeta_{l, \varsigma(k)}, \zeta_{l, \varsigma(k)}\right], \zeta_{l, \varsigma(k)} \triangleq \frac{1-\rho_{l, \varsigma(k)}}{1+\rho_{l, \varsigma(k)}}$, and $\rho_{l, \varsigma(k)} \triangleq e^{-\frac{2}{\varrho_{l, \varsigma(k)}}}(l=1,2, \ldots, m)$ with the Markovian jump quantization density $\varrho_{l, \varsigma(k)}$;

- for the Markovian packet losses [32], the model (6) is set as $\varsigma(k) \in\{1,2\}$, where the mode $\varsigma(k)=1$ means that the packet is received, i.e., $\Gamma_{1}=I_{m}, \Phi_{1}=0_{m}$, and the mode $\varsigma(k)=2$ implies that the packet is lost, i.e., $\Gamma_{2}=0_{m}, \Phi_{2}=0_{m}$.

Remark 2: Over the past decade, the SMC problem subject to actuator degradation has been addressed well as in [3], [45]. Unfortunately, in order to ensure the full column rank condition of control gain matrix under the actuator degradation, the actuator outage case is always excluded in the existing literature [3], [45]. In this paper, the fading model (6) covers the actuator outage as a special case in a stochastic setting, that is, the stochastic variable $\vartheta_{\varsigma(k)}(k)$ may be equal to zero at some sampling instants as shown in the simulation example later. This fact just shows a key contribution of this paper.

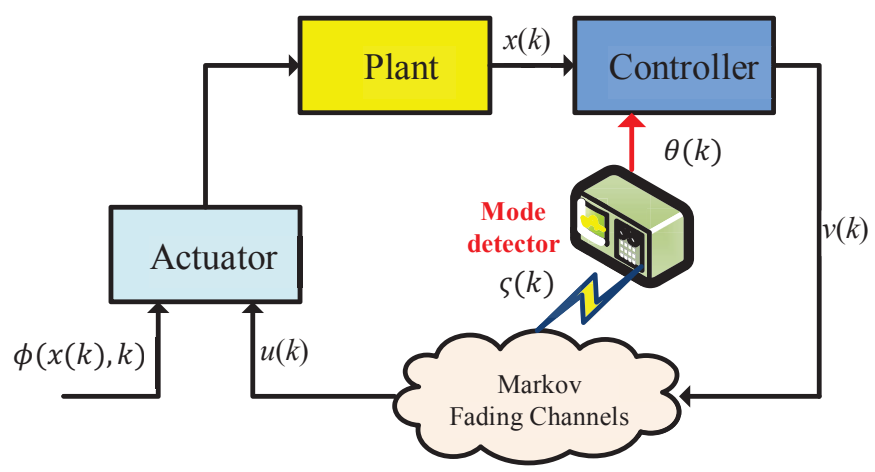

Fig. 2. Reliable SMC over hidden Markov fading channels

As depicted in Fig. 2, the mode detector is utilized to estimate the actual network mode $\varsigma(k)$ and also emit an estimated mode signal $\theta(k) \in \Xi \triangleq\{1,2, \ldots, L\}$ to the controller with the mode detection probability $\delta_{i q}$ given by

$$
\delta_{i q} \triangleq \operatorname{Pr}\{\theta(k)=q \mid \varsigma(k)=i\},
$$

where $\delta_{i q} \in[0,1]$ and $\sum_{q=1}^{L} \delta_{i q}=1$. We denote the mode detection probability matrix as $\Omega \triangleq\left[\delta_{i q}\right]_{i \in \Theta, q \in \Xi}$. It is easy to see that the detector (9) covers the following three special cases:

- Synchronous observation of network mode: $\Theta=\Xi$, and $\delta_{i i}=1(\theta(k)=\varsigma(k)$ in probability 1$) ;$

- Asynchronous observation of network mode: $\Xi \neq I$ $\left(\theta(k)=\varsigma(k)\right.$ with some probabilities $\left.0<\delta_{i i}<1\right)$;

- No observation/estimation of network mode: $L=1$ $(\theta(k)=1$ in probability 1$)$.

Remark 3: In fact, the asynchronous network mode observation can be interpreted as the multi-step delayed network mode observation in the sense of probability, where the mode detection probabilities $\left\{\delta_{i q}\right\}$ are obtained through the following statistical method via the channel field measurements [27]:

$$
\delta_{i q}=\lim _{n_{i} \rightarrow \infty} \frac{l_{i q}}{n_{i}}, i \in \Theta, q \in \Xi,
$$

where $n_{i} \in \mathbb{N}$ is the times of network mode $\varsigma(k)=i$, and $l_{i q} \in \mathbb{N}$ is the times of detector mode $\theta(k)=q$ under the 
case of system mode $\varsigma(k)=i$. The utilization of the hidden Markov mode detector (9) to the FSMFCs is motivated by [7], [7], [24], [37]. For presentation convenience, the novel FSMFCs (5)-(9) is named as the HMFCs hereafter.

In this paper, we are interested in developing a SMC input $v(k)$ by just using the estimated network mode signal $\theta(k)$ such that the resultant closed-loop system is mean-square stable subject to the HMFCs (5)-(9).

\section{MAIN RESULTS}

\section{A. Sliding function and sliding mode controller}

In this paper, the following linear sliding function is utilized:

$$
s(k)=T x(k)
$$

where $T \in \mathbb{R}^{m \times n}$ is a sliding gain matrix to be determined later such that

$$
\operatorname{det}(T B) \neq 0 \text {. }
$$

Remark 4: It is worth mentioning that the mode-dependent sliding surfaces as in [2], [17], [20], [21] may provide more freedom to design the mode-dependent controller. However, due to the switching frequently from one mode to another, the reachability of mode-dependent sliding surfaces may not always be attained actually. To this end, this paper utilizes the common sliding function (10).

By only employing the estimated mode signal $\theta(k) \in \Xi$ to the network mode $\varsigma(k)$, we construct the following SMC law for the state-saturated system (1):

$$
v(k)=K_{\theta(k)} x(k)-\varphi\|x(k)\| \cdot \operatorname{sgn}(s(k))
$$

where the matrices $K_{\theta(k)} \in \mathbb{R}^{m \times n}$ will be designed later.

Remark 5: It is well recognized that the chattering is the main drawback of the SMC approach. Notice that the matched external disturbance $\phi(x(k), k)$ in the system (1) is supposed to satisfy Lipchitz condition so that the discontinuous term can have an adaptive gain. This feature is actually benefit for chattering alleviation.

Combining (1), (6) and (12), we have the following closedloop system:

$$
x(k+1)=\sigma\left(\left(A+\Delta A(k)+B \vartheta_{i}(k) K_{q}\right) x(k)+B \varepsilon_{i}(k)\right),
$$

where $i \in \Theta, q \in \Xi$ and

$$
\varepsilon_{i}(k) \triangleq \phi(x(k), k)-\varphi\|x(k)\| \cdot \vartheta_{i}(k) \operatorname{sgn}(s(k)) .
$$

Definition 1: [5] The closed-loop system (13) is said to be mean-square stable if, for any initial condition $x(0) \neq 0$, the condition $\left.\lim _{k \rightarrow \infty} \mathbb{E}\left\{\|x(k)\|^{2}\right\}\right|_{x(0)}=0$ holds.

The following three technical lemmas will be useful in the subsequent investigation on the mean-square stability of the closed-loop system (13) and the reachability for the specified sliding surface (10).

Lemma 1: [8], [16] Let $\mathbb{Z}_{n}$ be the set of $n \times n$ diagonal matrices whose diagonal elements are either 1 or 0 . $i$ ) There are $2^{n}$ elements in $\mathbb{Z}_{n}$ where its $r$ th element is denoted as $Z_{r}$, $r \in \mathbb{M}_{n} \triangleq\left\{1,2 \ldots, 2^{n}\right\}$. ii) By defining $Z_{r}^{-} \triangleq I-Z_{r}$ and letting $G \in \mathbb{R}^{n \times n}$ satisfy $\|G\|_{\infty} \leq 1$, for any vector $w \in \mathbb{R}^{n}$, we have

$$
\begin{aligned}
& \Lambda^{-1} \sigma(A x(k)+w) \\
& \in \operatorname{co}\left\{Z_{r} \Lambda^{-1}(A x(k)+w)+Z_{r}^{-} G \Lambda^{-1} x(k)\right\}, r \in \mathbb{M}_{n}
\end{aligned}
$$

where the matrix $\Lambda$ is defined in (4), and $\operatorname{co}\{\cdot\}$ denotes the convex hull of a set.

Lemma 2: For any $\varsigma(k)=i \in \Theta$, the stochastic variable $\varepsilon_{i}(k)$ in (14) satisfies

$\mathbf{E}\left\{\varepsilon_{i}^{\mathrm{T}}(k) \varepsilon_{i}(k) \mid x(k)\right\} \leq\left(2+2\left\|\Gamma_{i}\right\|^{2}+\operatorname{tr}\left(\Psi_{i}\right)\right) \varphi^{2} x^{\mathrm{T}}(k) x(k)$.

Proof: For any $i \in \Theta$, it yields from (14) that

$$
\begin{aligned}
& \mathbf{E}\left\{\varepsilon_{i}^{\mathrm{T}}(k) \varepsilon_{i}(k) \mid x(k)\right\} \\
= & \|\phi(x(k), k)-\varphi\| x(k)\left\|\cdot \Gamma_{i} \operatorname{sgn}(s(k))\right\|^{2} \\
& +\varphi^{2}\|x(k)\|^{2} \operatorname{sgn}^{\mathrm{T}}(s(k)) \Psi_{i} \operatorname{sgn}(s(k)) \\
\leq & \left(2+2\left\|\Gamma_{i}\right\|^{2}+\operatorname{tr}\left(\Psi_{i}\right)\right) \varphi^{2} x^{\mathrm{T}}(k) x(k),
\end{aligned}
$$

which completes the proof.

Lemma 3: Let $X, Y$ and $Z$ be positive semidefinite matrices. If $X \leq Y$, then the inequality $Z \circ X \leq Z \circ Y$ holds.

Proof: Clearly, $Y-X \geq 0$. Then, according to Theorem 7.5.3 in [12], we have $Z \circ(Y-X) \geq 0$, which is equivalent to $Z \circ X \leq Z \circ Y$. The proof is complete.

\section{B. Analysis of sliding mode dynamics}

In the following theorem, we analyze the mean-square stability of the closed-loop system (13) by utilizing a fadingrelated state-dependent Lyapunov function.

Theorem 1: Consider the networked state-saturated system (1) and the SMC law (12). The closed-loop system (13) is mean-square stable if, for any $i \in \Theta, q \in \Xi$ and $r \in \mathbb{M}_{n}$, there exist matrices $P_{i}>0, K_{q} \in \mathbb{R}^{m \times n}, G \in \mathbb{R}^{n \times n}$, and scalars $\gamma_{i r}>0$ such that the following matrix inequalities hold:

$$
\begin{aligned}
& \|G\|_{\infty} \leq 1, \\
& B^{\mathrm{T}} \Lambda^{-1} Z_{r} \Lambda \mathbb{P}_{i} \Lambda Z_{r} \Lambda^{-1} B \leq \gamma_{i r} I, \\
& -P_{i}+2 \gamma_{i r}\left(2+2\left\|\Gamma_{i}\right\|^{2}+\operatorname{tr}\left(\Psi_{i}\right)\right) \varphi^{2} I \\
& +2 \sum_{q=1}^{L} \delta_{i q}\left\{\mathbf{Z}_{i r q}^{\mathrm{T}} \Lambda \mathbb{P}_{i} \Lambda \mathbf{Z}_{i r q}\right. \\
& \left.+K_{q}^{\mathrm{T}}\left(\Phi_{i} \circ B^{\mathrm{T}} \Lambda^{-1} Z_{r} \Lambda \mathbb{P}_{i} \Lambda Z_{r} \Lambda^{-1} B\right) K_{q}\right\}<0,
\end{aligned}
$$

where $\mathbb{P}_{i} \triangleq \sum_{j=1}^{N} \pi_{i j} P_{j}$, and $\mathbf{Z}_{i r q} \triangleq Z_{r} \Lambda^{-1}(A+\Delta A(k))+$ $Z_{r}^{-} G \Lambda^{-1}+Z_{r} \Lambda^{-1} B \Gamma_{i} K_{q}$.

Proof: For the network mode $\varsigma(k)=i \in \Theta$, we consider the following Lyapunov function:

$$
V(x(k), \varsigma(k)) \triangleq x^{\mathrm{T}}(k) P_{\varsigma(k)} x(k) .
$$

Exploiting the conditional expectation [41] and Lemma 1, we have the following relationships for $\varsigma(k)=i$ :

$$
\begin{aligned}
& \mathbf{E}\{\Delta V(x(k), \varsigma(k)) \mid x(k)\} \\
\triangleq & \mathbf{E}\left\{x^{\mathrm{T}}(k+1) P_{\varsigma(k+1)} x(k+1) \mid x(k)\right\}
\end{aligned}
$$




$$
\begin{aligned}
& -x^{\mathrm{T}}(k) P_{i} x(k) \\
= & \mathbf{E}\left\{x^{\mathrm{T}}(k+1) \mathbb{P}_{i} x(k+1) \mid x(k)\right\}-x^{\mathrm{T}}(k) P_{i} x(k) \\
= & \sum_{q=1}^{L} \delta_{i q} \mathbf{E}\left\{\sigma^{\mathrm{T}}\left(\left(A+\Delta A(k)+B \vartheta_{i}(k) K_{q}\right) x(k)+B \varepsilon_{i}(k)\right)\right. \\
& \left.\times \mathbb{P}_{i} \sigma\left(\left(A+\Delta A(k)+B \vartheta_{i}(k) K_{q}\right) x(k)+B \varepsilon_{i}(k)\right) \mid x(k)\right\} \\
& -x^{\mathrm{T}}(k) P_{i} x(k) \\
= & \sum_{q=1}^{L} \delta_{i q} \mathbf{E}\left\{\left\{\sum _ { r = 1 } ^ { 2 ^ { n } } \alpha _ { r } \left[Z_{r} \Lambda^{-1}((A+\Delta A(k)\right.\right.\right. \\
& \left.\left.\left.\left.+B \vartheta_{i}(k) K_{q}\right) x(k)+B \varepsilon_{i}(k)\right)+Z_{r}^{-} G \Lambda^{-1} x(k)\right]^{\mathrm{T}}\right\} \\
& \times \Lambda \mathbb{P}_{i} \Lambda\left\{\sum _ { r = 1 } ^ { 2 ^ { n } } \alpha _ { r } \left[Z_{r} \Lambda^{-1}((A+\Delta A(k)\right.\right. \\
& \left.\left.\left.\left.\left.+B \vartheta_{i}(k) K_{q}\right) x(k)+B \varepsilon_{i}(k)\right)+Z_{r}^{-} G \Lambda^{-1} x(k)\right]\right\} \mid x(k)\right\} \\
& -x^{\mathrm{T}}(k) P_{i} x(k) \\
& +x_{r \in \mathbb{M}_{n}}\left\{\sum _ { q = 1 } ^ { L } \delta _ { i q } \mathbf { E } \left\{\left[Z_{r} \Lambda^{-1}\left((A) P_{i} x(k)\right\}\right.\right.\right. \\
& \left.\left.+Z_{r} \Lambda^{-1} B \vartheta_{i}(k) K_{q}\right] x(k) \mid x(k)\right\} \\
& \left.\left.\left.\left.+B \vartheta_{i}(k) K_{q}\right) x(k)+B \varepsilon_{i}(k)\right)+Z_{r}^{-} G \Lambda^{-1} x(k) B^{\mathrm{T}} \Lambda^{-1} Z_{r} \Lambda \mathbb{P}_{i} \Lambda Z_{r} \Lambda^{-1} B \varepsilon_{i}(k) \mid x(k)\right\}\right\} \\
& \left.+Z_{r}^{-} G \Lambda^{-1}+Z_{r} \Lambda^{-1} B \vartheta_{i}(k) K_{q}\right]^{\mathrm{T}} \\
& \times \mathbb{M}_{i} \Lambda\left[Z_{r} \Lambda^{-1}((A+\Delta A(k)\right. \\
& \left.\left.\left.\left.\left.+B \vartheta_{i}(k) K_{q}\right) x(k)+B \varepsilon_{i}(k)\right)+Z_{r}^{-} G \Lambda^{-1} x(k)\right] \mid x(k)\right\}\right\} \\
& -Z_{r} \Lambda_{i}^{-1}(A(k) \\
& \max _{i q}\left\{2 \mathbf { E } \left\{x ^ { \mathrm { T } } ( k ) \left[Z_{r} \Lambda^{-1}(A+\Delta A(k))\right.\right.\right. \\
& \\
& \\
&
\end{aligned}
$$

where $\alpha_{r} \geq 0$ and $\sum_{r=1}^{2^{n}} \alpha_{r}=1$.

It follows from (16) and Lemma 2 that

$$
\begin{aligned}
& \mathbf{E}\left\{\varepsilon_{i}^{\mathrm{T}}(k) B^{\mathrm{T}} \Lambda^{-1} Z_{r} \Lambda \mathbb{P}_{i} \Lambda Z_{r} \Lambda^{-1} B \varepsilon_{i}(k) \mid x(k)\right\} \\
\leq & \gamma_{i r}\left(2+2\left\|\Gamma_{i}\right\|^{2}+\operatorname{tr}\left(\Psi_{i}\right)\right) \varphi^{2} x^{\mathrm{T}}(k) x(k) .
\end{aligned}
$$

Then, for $\varsigma(k)=i$, substituting (20) into (19) gives

$$
\mathbf{E}\{\Delta V(x(k), \varsigma(k)) \mid x(k)\}
$$

$$
\begin{aligned}
\leq & \max _{r \in \mathbb{M}_{n}}\left\{\sum _ { q = 1 } ^ { L } \delta _ { i q } \left\{2 \left\{x ^ { \mathrm { T } } ( k ) \left[Z_{r} \Lambda^{-1}(A+\Delta A(k))\right.\right.\right.\right. \\
& \left.+Z_{r}^{-} G \Lambda^{-1}+Z_{r} \Lambda^{-1} B \Gamma_{i} K_{q}\right]^{\mathrm{T}} \\
& \times \Lambda \mathbb{P}_{i} \Lambda\left[Z_{r} \Lambda^{-1}(A+\Delta A(k))+Z_{r}^{-} G \Lambda^{-1}\right. \\
& \left.+Z_{r} \Lambda^{-1} B \Gamma_{i} K_{q}\right] x(k) \\
& \left.+x^{\mathrm{T}}(k) K_{q}^{\mathrm{T}}\left(\Phi_{i} \circ B^{\mathrm{T}} \Lambda^{-1} Z_{r} \Lambda \mathbb{P}_{i} \Lambda Z_{r} \Lambda^{-1} B\right) K_{q} x(k)\right\} \\
& \left.+2 \gamma_{i r}\left(2+2\left\|\Gamma_{i}\right\|^{2}+\operatorname{tr}\left(\Psi_{i}\right)\right) \varphi^{2} x^{\mathrm{T}}(k) x(k)\right\} \\
& \left.-x^{\mathrm{T}}(k) P_{i} x(k)\right\} .
\end{aligned}
$$

It is easily shown that, for any $\varsigma(k)=i \in \Theta$, $\mathbf{E}\{\Delta V(x(k), i) \mid x(k)\}<0$ if the condition (17) holds, and this completes the proof.

Remark 6: In the discrete-time setting of the SMC theory, the equivalent control law has been widely employed to analyze the stability of the sliding mode dynamics, see [23], [32], [33] for example. Unfortunately, it follows from (1), (6) and (10) that

$$
\begin{aligned}
s(k+1)= & T \sigma((A+\Delta A(k)) x(k) \\
& \left.+B\left(\vartheta_{\varsigma(k)}(k) v(k)+\phi(x(k), k)\right)\right),
\end{aligned}
$$

from which one can see that the equivalent control law approach cannot be employed in this paper due to the coexistence of the state-saturation function $\sigma(\cdot)$ and the channel fading variable $\vartheta_{\varsigma(k)}(k)$, and this gives rise to substantial challenges regarding the stability analysis of the SMC systems subject to state saturation and HMFCs. With the aid of Lemmas 1 and 2 as well as the HMM approach, sufficient conditions are established such that the closed-loop system (13) is mean-square stable under the SMC law (12).

\section{Analysis of reachability}

This subsection is devoted to the analysis of the reachability for the specified sliding surface (10). First, it follows from (10) and (13) that

$$
s(k+1)=T \sigma\left(\left(A+\Delta A(k)+B \vartheta_{i}(k) K_{q}\right) x(k)+B \varepsilon_{i}(k)\right) .
$$

Next, define the following time-varying sliding domain:

$$
\mathcal{O} \triangleq\{s(k) \mid\|s(k)\| \leq \tilde{\epsilon}(k)\}
$$

where

$$
\begin{aligned}
\tilde{\epsilon}(k) \triangleq & \max _{i \in \Theta, r \in \mathbb{M}_{n}}\left\{\sqrt{\frac{\epsilon_{i r}(k)}{\lambda_{\min }\left(W_{i}\right)}}\right\}, \\
\epsilon_{i r}(k) \triangleq & {\left[\lambda_{\max }\left(B^{\mathrm{T}} \Lambda^{-1} Z_{r} \Lambda \mathbb{P}_{i} \Lambda Z_{r} \Lambda^{-1} B\right)\right.} \\
& \left.+\lambda_{\max }\left(B^{\mathrm{T}} \Lambda^{-1} Z_{r} \Lambda T^{\mathrm{T}} \mathbb{W}_{i} T \Lambda Z_{r} \Lambda^{-1} B\right)\right] \\
& \times\left(2+2\left\|\Gamma_{i}\right\|^{2}+\operatorname{tr}\left(\Psi_{i}\right)\right) \varphi^{2}\|x(k)\|^{2}
\end{aligned}
$$


with the matrix $\mathbb{P}_{i}$ being defined in Theorem 1 , and the matrices $W_{i}$ and $\mathbb{W}_{i}$ being defined in Theorem 2.

Clearly, $\mathcal{O}$ is just a vicinity of the sliding surface (10), i.e., the quasi-sliding mode (QSM) around the sliding surface $s(k)=0$. In the following theorem, we will propose a sufficient condition to ensure the above QSM motion for the state-saturated system (1) subject to the HMFCs (5)-(9).

Theorem 2: Consider the networked state-saturated system (1) and the SMC law (12). For any $i \in \Theta, q \in \Xi$ and $r \in \mathbb{M}_{n}$, assume that there exist matrices $P_{i}>0, W_{i}>0, K_{q} \in \mathbb{R}^{m \times n}$, $T \in \mathbb{R}^{m \times n}$ and $G \in \mathbb{R}^{n \times n}$ satisfying the condition (15) and the following matrix inequalities:

$$
\begin{aligned}
& -P_{i}+2 \sum_{q=1}^{L} \delta_{i q}\left\{\mathbf{Z}_{i r q}^{\mathrm{T}} \Lambda \mathbb{P}_{i} \Lambda \mathbf{Z}_{i r q}+\mathbf{Z}_{i r q}^{\mathrm{T}} \Lambda T^{\mathrm{T}} \mathbb{W}_{i} T \Lambda \mathbf{Z}_{i r q}\right. \\
& +K_{q}^{\mathrm{T}}\left(\Phi_{i} \circ B^{\mathrm{T}} \Lambda^{-1} Z_{r} \Lambda \mathbb{P}_{i} \Lambda Z_{r} \Lambda^{-1} B\right) K_{q} \\
& \left.+K_{q}^{\mathrm{T}}\left(\Phi_{i} \circ B^{\mathrm{T}} \Lambda^{-1} Z_{r} \Lambda T^{\mathrm{T}} \mathbb{W}_{i} T \Lambda Z_{r} \Lambda^{-1} B\right) K_{q}\right\}<0
\end{aligned}
$$

where $\mathbb{W}_{i} \triangleq \sum_{j=1}^{N} \pi_{i j} W_{j}$, and $\mathbb{P}_{i}, \mathbf{Z}_{i r q}$ are defined in Theorem 1. Then, the SMC law (12) can force the state trajectories of the closed-loop system (13) into the sliding region $\mathcal{O}$ in mean-square sense.

Proof: Consider the following extended Lyapunov functional:

$$
\tilde{V}(x(k), \varsigma(k)) \triangleq x^{\mathrm{T}}(k) P_{\varsigma(k)} x(k)+s^{\mathrm{T}}(k) W_{\varsigma(k)} s(k) .
$$

Along with the solution of the sliding function (22), for any $\varsigma(k)=i \in \Theta$, one has from Lemma 1 that

$$
\mathbf{E}\left\{s^{\mathrm{T}}(k+1) W(\varsigma(k+1)) s(k+1) \mid x(k)\right\}
$$$$
=\sum_{q=1}^{L} \delta_{i q} \mathbf{E}\left\{\left\{\sum _ { r = 1 } ^ { 2 ^ { n } } \alpha _ { r } \left[Z_{r} \Lambda^{-1}((A+\Delta A(k)\right.\right.\right.
$$$$
\left.\left.\left.\left.+B \vartheta_{i}(k) K_{q}\right) x(k)+B \varepsilon_{i}(k)\right)+Z_{r}^{-} G \Lambda^{-1} x(k)\right]^{\mathrm{T}}\right\}
$$$$
\times \Lambda T^{\mathrm{T}} \mathbb{W}_{i} T \Lambda\left\{\sum _ { r = 1 } ^ { 2 ^ { n } } \alpha _ { r } \left[Z_{r} \Lambda^{-1}((A+\Delta A(k)\right.\right.
$$$$
\left.\left.\left.\left.\left.+B \vartheta_{i}(k) K_{q}\right) x(k)+B \varepsilon_{i}(k)\right)+Z_{r}^{-} G \Lambda^{-1} x(k)\right]\right\} \mid x(k)\right\}
$$$$
\leq \max _{r \in \mathbb{M}_{n}}\left\{\sum _ { q = 1 } ^ { L } \delta _ { i q } \left\{2 \mathbf { E } \left\{x ^ { \mathrm { T } } ( k ) \left[Z_{r} \Lambda^{-1}(A+\Delta A(k))\right.\right.\right.\right.
$$

$$
\left.+Z_{r}^{-} G \Lambda^{-1}+Z_{r} \Lambda^{-1} B \vartheta_{i}(k) K_{q}\right]^{\mathrm{T}}
$$$$
\times \Lambda T^{\mathrm{T}} \mathbb{W}_{i} T \Lambda\left[Z_{r} \Lambda^{-1}(A+\Delta A(k))+Z_{r}^{-} G \Lambda^{-1}\right.
$$$$
\left.\left.+Z_{r} \Lambda^{-1} B \vartheta_{i}(k) K_{q}\right] x(k) \mid x(k)\right\}
$$$$
\left.\left.+2 \mathbf{E}\left\{\varepsilon_{i}^{\mathrm{T}}(k) B^{\mathrm{T}} \Lambda^{-1} Z_{r} \Lambda T^{\mathrm{T}} \mathbb{W}_{i} T \Lambda Z_{r} \Lambda^{-1} B \varepsilon_{i}(k) \mid x(k)\right\}\right\}\right\}
$$

$$
\begin{aligned}
\leq & \max _{r \in \mathbb{M}_{n}}\left\{\sum _ { q = 1 } ^ { L } \delta _ { i q } \left\{2 \left\{x ^ { \mathrm { T } } ( k ) \left[Z_{r} \Lambda^{-1}(A+\Delta A(k))\right.\right.\right.\right. \\
& \left.+Z_{r}^{-} G \Lambda^{-1}+Z_{r} \Lambda^{-1} B \Gamma_{i} K_{q}\right]^{\mathrm{T}}
\end{aligned}
$$

$$
\begin{aligned}
& \times \Lambda T^{\mathrm{T}} \mathbb{W}_{i} T \Lambda\left[Z_{r} \Lambda^{-1}(A+\Delta A(k))+Z_{r}^{-} G \Lambda^{-1}\right. \\
& \left.+Z_{r} \Lambda^{-1} B \Gamma_{i} K_{q}\right] x(k)+x^{\mathrm{T}}(k) K_{q}^{\mathrm{T}} \\
& \left.\times\left(\Phi_{i} \circ B^{\mathrm{T}} \Lambda^{-1} Z_{r} \Lambda T^{\mathrm{T}} \mathbb{W}_{i} T \Lambda Z_{r} \Lambda^{-1} B\right) K_{q} x(k)\right\} \\
& +2 \lambda_{\max }\left(B^{\mathrm{T}} \Lambda^{-1} Z_{r} \Lambda T^{\mathrm{T}} \mathbb{W}_{i} T \Lambda Z_{r} \Lambda^{-1} B\right) \\
& \left.\left.\times\left(2+2\left\|\Gamma_{i}\right\|^{2}+\operatorname{tr}\left(\Psi_{i}\right)\right) \varphi^{2}\|x(k)\|^{2}\right\}\right\},
\end{aligned}
$$

where $\alpha_{r} \geq 0$ and $\sum_{r=1}^{2^{n}} \alpha_{r}=1$.

Bearing (19) and (26) in mind, we obtain the following relationships for any $\varsigma(k)=i \in \Theta$ :

$$
\begin{aligned}
\mathbf{E} & \{\Delta \tilde{V}(x(k), \varsigma(k)) \mid x(k)\} \\
\triangleq & \mathbf{E}\{\Delta V(x(k), \varsigma(k)) \mid x(k)\} \\
& +\mathbf{E}\left\{s^{\mathrm{T}}(k+1) W(\varsigma(k+1)) s(k+1) \mid x(k)\right\} \\
& -s^{\mathrm{T}}(k) W_{\varsigma(k)} s(k) \\
\leq & \max _{r \in \mathbb{M}_{n}}\left\{\sum _ { q = 1 } ^ { L } \delta _ { i q } \left\{2 \left\{x ^ { \mathrm { T } } ( k ) \left[Z_{r} \Lambda^{-1}(A+\Delta A(k))\right.\right.\right.\right. \\
& \left.+Z_{r}^{-} G \Lambda^{-1}+Z_{r} \Lambda^{-1} B \Gamma_{i} K_{q}\right]^{\mathrm{T}} \\
& \times \Lambda \mathbb{P}_{i} \Lambda\left[Z_{r} \Lambda^{-1}(A+\Delta A(k))+Z_{r}^{-} G \Lambda^{-1}\right. \\
& \left.+Z_{r} \Lambda^{-1} B \Gamma_{i} K_{q}\right] x(k) \\
& \left.+x^{\mathrm{T}}(k) K_{q}^{\mathrm{T}}\left(\Phi_{i} \circ B^{\mathrm{T}} \Lambda^{-1} Z_{r} \Lambda \mathbb{P}_{i} \Lambda Z_{r} \Lambda^{-1} B\right) K_{q} x(k)\right\} \\
& +2\left\{x ^ { \mathrm { T } } ( k ) \left[Z_{r} \Lambda^{-1}(A+\Delta A(k))\right.\right. \\
& \left.+Z_{r}^{-} G \Lambda^{-1}+Z_{r} \Lambda^{-1} B \Gamma_{i} K_{q}\right]^{\mathrm{T}} \\
& \times \Lambda T^{\mathrm{T}} \mathbb{W}_{i} T \Lambda\left[Z_{r} \Lambda^{-1}(A+\Delta A(k))+Z_{r}^{-} G \Lambda^{-1}\right. \\
& \left.+Z_{r} \Lambda^{-1} B \Gamma_{i} K_{q}\right] x(k)+x^{\mathrm{T}}(k) K_{q}^{\mathrm{T}} \\
& \left.\left.\times\left(\Phi_{i} \circ B^{\mathrm{T}} \Lambda^{-1} Z_{r} \Lambda T^{\mathrm{T}} \mathbb{W}_{i} T \Lambda Z_{r} \Lambda^{-1} B\right) K_{q} x(k)\right\}\right\} \\
& \left.-x^{\mathrm{T}}(k) P_{i} x(k)-\lambda_{\min }\left(W_{i}\right)\|s(k)\|^{2}+\epsilon_{i r}(k)\right\} .
\end{aligned}
$$

If the following is true:

$$
\|s(k)\|>\tilde{\epsilon}(k) \geq \sqrt{\frac{\epsilon_{i r}(k)}{\lambda_{\min }\left(W_{i}\right)}}, \forall i \in \Theta, r \in \mathbb{M}_{n},
$$

that is, the state trajectories escape the region $\mathcal{O}$, then we can have from (24) and (27) that

$$
\begin{aligned}
& \mathbf{E}\{\Delta \tilde{V}(x(k), \varsigma(k)) \mid x(k)\} \\
& \leq \max _{r \in \mathbb{M}_{n}}\left\{\sum _ { q = 1 } ^ { L } \delta _ { i q } \left\{2 \left\{x ^ { \mathrm { T } } ( k ) \left[Z_{r} \Lambda^{-1}(A+\Delta A(k))\right.\right.\right.\right. \\
& \left.+Z_{r}^{-} G \Lambda^{-1}+Z_{r} \Lambda^{-1} B \Gamma_{i} K_{q}\right]^{\mathrm{T}} \\
& \quad \times \Lambda \mathbb{P}_{i} \Lambda\left[Z_{r} \Lambda^{-1}(A+\Delta A(k))+Z_{r}^{-} G \Lambda^{-1}\right.
\end{aligned}
$$




$$
\begin{aligned}
& \left.+Z_{r} \Lambda^{-1} B \Gamma_{i} K_{q}\right] x(k) \\
& \left.+x^{\mathrm{T}}(k) K_{q}^{\mathrm{T}}\left(\Phi_{i} \circ B^{\mathrm{T}} \Lambda^{-1} Z_{r} \Lambda \mathbb{P}_{i} \Lambda Z_{r} \Lambda^{-1} B\right) K_{q} x(k)\right\} \\
& +2\left\{x ^ { \mathrm { T } } ( k ) \left[Z_{r} \Lambda^{-1}(A+\Delta A(k))\right.\right. \\
& \left.+Z_{r}^{-} G \Lambda^{-1}+Z_{r} \Lambda^{-1} B \Gamma_{i} K_{q}\right]^{\mathrm{T}} \\
& \times \Lambda T^{\mathrm{T}} \mathbb{W}_{i} T \Lambda\left[Z_{r} \Lambda^{-1}(A+\Delta A(k))+Z_{r}^{-} G \Lambda^{-1}\right. \\
& \left.+Z_{r} \Lambda^{-1} B \Gamma_{i} K_{q}\right] x(k)+x^{\mathrm{T}}(k) K_{q}^{\mathrm{T}} \\
& \left.\left.\times\left(\Phi_{i} \circ B^{\mathrm{T}} \Lambda^{-1} Z_{r} \Lambda T^{\mathrm{T}} \mathbb{W}_{i} T \Lambda Z_{r} \Lambda^{-1} B\right) K_{q} x(k)\right\}\right\} \\
& \left.-x^{\mathrm{T}}(k) P_{i} x(k)\right\} \\
& <0, \forall i \in \Theta
\end{aligned}
$$

which means that, outside the region $\mathcal{O}$ defined in (23), the state trajectories of the closed-loop system (13) are strictly deceasing in mean-square sense. The proof is now complete.

\section{Synthesis of SMC law}

Actually, it is quite difficult to directly solve the inequalities in Theorems 1 and 2 due to the coupling terms and the Hadamard product terms in (17) and (24). In the following theorem, by means of the property of the Hadamard product in Lemma 3, we establish a sufficient condition to guarantee the mean-square stability of the closed-loop system (13) and the reachability of the specified sliding surface (10) simultaneously.

Theorem 3: For any $i \in \Theta, q \in \Xi$ and $r \in \mathbb{M}_{n}$, assume that there exist $\bar{P}_{i}>0, \bar{W}_{i}>0, \bar{Q}_{i r q}>0, \mathcal{K}_{q} \in \mathbb{R}^{m \times n}, J_{q} \in$ $\mathbb{R}^{n \times n}, T \in \mathbb{R}^{m \times n}, G \in \mathbb{R}^{n \times n}$, and scalars $\bar{\gamma}_{i r}>0, \bar{\zeta}_{i r}>0$, $\chi>0$ such that the following coupled matrix inequalities hold:

$$
\begin{aligned}
& \|G\|_{\infty} \leq 1 \\
& {\left[\begin{array}{cc}
-\bar{\gamma}_{i r} I & \mathcal{B}_{i r} \\
\mathcal{B}_{i r}^{\mathrm{T}} & -\mathcal{P}
\end{array}\right] \leq 0,} \\
& {\left[\begin{array}{cc}
-\bar{\zeta}_{i r} I & \mathcal{T}_{i r} \\
\mathcal{T}_{i r}^{\mathrm{T}} & -\mathcal{W}
\end{array}\right] \leq 0,} \\
& {\left[\begin{array}{cc}
\tilde{\mathcal{Q}}_{i r q} & \Sigma_{i r q} \\
\Sigma_{i r q}^{\mathrm{T}} & -\mathcal{I}_{i r}
\end{array}\right] \leq 0,} \\
& {\left[\begin{array}{ccc}
-\bar{P}_{i} & \tilde{\varphi}_{i} \bar{P}_{i} & \tilde{\mathcal{P}}_{i} \\
\tilde{\varphi}_{i} \bar{P}_{i} & -\bar{\gamma}_{i r} I & 0 \\
\tilde{\mathcal{P}}_{i} & 0 & -\mathcal{Q}_{i r}
\end{array}\right]<0,}
\end{aligned}
$$

where

$$
\begin{aligned}
& \mathcal{P} \triangleq \operatorname{diag}\left\{\bar{P}_{1}, \bar{P}_{2}, \ldots, \bar{P}_{N}\right\}, \mathcal{W} \triangleq \operatorname{diag}\left\{\bar{W}_{1}, \bar{W}_{2}, \ldots, \bar{W}_{N}\right\}, \\
& \mathcal{B}_{i r} \triangleq\left[\begin{array}{lll}
\bar{\gamma}_{i r} \sqrt{\pi_{i 1}} B^{\mathrm{T}} \Lambda^{-1} Z_{r} \Lambda \quad \ldots \quad & \bar{\gamma}_{i r} \sqrt{\pi_{i N}} B^{\mathrm{T}} \Lambda^{-1} Z_{r} \Lambda
\end{array}\right], \\
& \mathcal{T}_{i r} \triangleq\left[\begin{array}{lll}
\bar{\zeta}_{i r} \sqrt{\pi_{i 1}} B^{\mathrm{T}} \Lambda^{-1} Z_{r} \Lambda T^{\mathrm{T}} & \ldots \\
& \bar{\zeta}_{i r} \sqrt{\pi_{i N}} B^{\mathrm{T}} \Lambda^{-1} Z_{r} \Lambda T^{\mathrm{T}}
\end{array}\right], \\
& \tilde{\varphi}_{i} \triangleq \varphi \sqrt{2\left(2+2\left\|\Gamma_{i}\right\|^{2}+\operatorname{tr}\left(\Psi_{i}\right)\right)},
\end{aligned}
$$

$$
\begin{aligned}
& \tilde{\mathcal{P}}_{i} \triangleq\left[\begin{array}{llll}
\sqrt{2 \delta_{i 1}} \bar{P}_{i} & \sqrt{2 \delta_{i 2}} \bar{P}_{i} & \cdots & \sqrt{2 \delta_{i L}} \bar{P}_{i}
\end{array}\right], \\
& \mathcal{Q}_{i r} \triangleq \operatorname{diag}\left\{\bar{Q}_{i r 1}, \bar{Q}_{i r 2}, \ldots, \bar{Q}_{i r L}\right\} \text {, } \\
& \tilde{\mathcal{Q}}_{i r q} \triangleq\left[\begin{array}{ccc}
\bar{Q}_{i r q}-J_{q}^{\mathrm{T}}-J_{q} & \mathcal{Z}_{i r q} & \tilde{\mathcal{Z}}_{i r q} \\
\mathcal{Z}_{i r q}^{\mathrm{T}} & -\mathcal{P} & 0 \\
\tilde{\mathcal{Z}}_{i r q}^{\mathrm{T}} & 0 & -\mathcal{W}
\end{array}\right] \text {, } \\
& \Sigma_{i r q} \triangleq\left[\begin{array}{cccc}
\mathcal{K}_{q}^{\mathrm{T}} \Psi_{i} & \mathcal{K}_{q}^{\mathrm{T}} \Psi_{i} & J_{q}^{\mathrm{T}} N^{\mathrm{T}} & 0 \\
0 & 0 & 0 & \chi \mathcal{M}_{i r} \\
0 & 0 & 0 & \chi \tilde{\mathcal{M}}_{i r}
\end{array}\right] \text {, } \\
& \mathcal{I}_{i r} \triangleq \operatorname{diag}\left\{\bar{\gamma}_{i r} \Psi_{i}, \bar{\zeta}_{i r} \Psi_{i}, \chi I, \chi I\right\} \text {, } \\
& \mathcal{Z}_{i r q} \triangleq\left[\begin{array}{llll}
\sqrt{\pi_{i 1}} \tilde{\mathbf{Z}}_{i r q} & \sqrt{\pi_{i 2}} \tilde{\mathbf{Z}}_{i r q} & \cdots & \sqrt{\pi_{i N}} \tilde{\mathbf{Z}}_{i r q}
\end{array}\right], \\
& \tilde{\mathbf{Z}}_{i r q} \triangleq\left(Z_{r} \Lambda^{-1} A J_{q}+Z_{r}^{-} G \Lambda^{-1} J_{q}+Z_{r} \Lambda^{-1} B \Gamma_{i} \mathcal{K}_{q}\right)^{\mathrm{T}} \Lambda, \\
& \tilde{\mathcal{Z}}_{i r q} \triangleq\left[\begin{array}{llll}
\sqrt{\pi_{i 1}} \overrightarrow{\mathbf{Z}}_{i r q} & \sqrt{\pi_{i 2}} \overrightarrow{\mathbf{Z}}_{i r q} & \cdots & \sqrt{\pi_{i N}} \overrightarrow{\mathbf{Z}}_{i r q}
\end{array}\right], \\
& \overrightarrow{\mathbf{Z}}_{i r q} \triangleq\left(Z_{r} \Lambda^{-1} A J_{q}+Z_{r}^{-} G \Lambda^{-1} J_{q}+Z_{r} \Lambda^{-1} B \Gamma_{i} \mathcal{K}_{q}\right)^{\mathrm{T}} \Lambda T^{\mathrm{T}}, \\
& \mathcal{M}_{i r} \triangleq\left[\begin{array}{lll}
\sqrt{\pi_{i 1}} M^{\mathrm{T}} \Lambda^{-1} Z_{r} \Lambda & \cdots & \sqrt{\pi_{i N}} M^{\mathrm{T}} \Lambda^{-1} Z_{r} \Lambda
\end{array}\right]^{\mathrm{T}}, \\
& \tilde{\mathcal{M}}_{i r} \triangleq\left[\sqrt{\pi_{i 1}} M^{\mathrm{T}} \Lambda^{-1} Z_{r} \Lambda T^{\mathrm{T}} \quad \ldots\right. \\
& \left.\sqrt{\pi_{i N}} M^{\mathrm{T}} \Lambda^{-1} Z_{r} \Lambda T^{\mathrm{T}}\right]^{\mathrm{T}} \text {. }
\end{aligned}
$$

Then, the SMC law (12) with $K_{q}=\mathcal{K}_{q} J_{q}^{-1}$ can guarantee both the mean-square stability of the closed-loop system (13) and the QSM with the sliding region $\mathcal{O}$ define in (23).

Proof: For any $i \in \Theta$ and $r \in \mathbb{M}_{n}$, we consider the following inequalities:

$$
\begin{aligned}
& \|G\|_{\infty} \leq 1 \\
& B^{\mathrm{T}} \Lambda^{-1} Z_{r} \Lambda \mathbb{P}_{i} \Lambda Z_{r} \Lambda^{-1} B \leq \gamma_{i r} I \\
& B^{\mathrm{T}} \Lambda^{-1} Z_{r} \Lambda T^{\mathrm{T}} \mathbb{W}_{i} T \Lambda Z_{r} \Lambda^{-1} B \leq \zeta_{i r} I \\
& -P_{i}+2 \gamma_{i r}\left(2+2\left\|\Gamma_{i}\right\|^{2}+\operatorname{tr}\left(\Psi_{i}\right)\right) \varphi^{2} I \\
& +2 \sum_{q=1}^{L} \delta_{i q}\left\{\mathbf{Z}_{i r q}^{\mathrm{T}} \Lambda \mathbb{P}_{i} \Lambda \mathbf{Z}_{i r q}+\mathbf{Z}_{i r q}^{\mathrm{T}} \Lambda T^{\mathrm{T}} \mathbb{W}_{i} T \Lambda \mathbf{Z}_{i r q}\right. \\
& +K_{q}^{\mathrm{T}}\left(\Phi_{i} \circ B^{\mathrm{T}} \Lambda^{-1} Z_{r} \Lambda \mathbb{P}_{i} \Lambda Z_{r} \Lambda^{-1} B\right) K_{q} \\
& \left.+K_{q}^{\mathrm{T}}\left(\Phi_{i} \circ B^{\mathrm{T}} \Lambda^{-1} Z_{r} \Lambda T^{\mathrm{T}} \mathbb{W}_{i} T \Lambda Z_{r} \Lambda^{-1} B\right) K_{q}\right\}<0 .
\end{aligned}
$$

It is straightforward to find that the conditions (17) and (24) are guaranteed by the inequality (37), which implies that Theorems 1 and 2 are ensured by the inequalities (34), (35) and (37).

By using Lemma 3 and the definition of the Hadamard product, it follows from the inequalities (35) and (36) that

$$
\begin{aligned}
& \Phi_{i} \circ B^{\mathrm{T}} \Lambda^{-1} Z_{r} \Lambda \mathbb{P}_{i} \Lambda Z_{r} \Lambda^{-1} B \leq \Phi_{i} \circ \gamma_{i r} I=\gamma_{i r} \Psi_{i}, \\
& \Phi_{i} \circ B^{\mathrm{T}} \Lambda^{-1} Z_{r} \Lambda T^{\mathrm{T}} \mathbb{W}_{i} T \Lambda Z_{r} \Lambda^{-1} B \leq \Phi_{i} \circ \zeta_{i r} I=\zeta_{i r} \Psi_{i} .
\end{aligned}
$$

Thus, the inequality (37) is ensured by

$$
\begin{aligned}
& -P_{i}+2 \gamma_{i r}\left(2+2\left\|\Gamma_{i}\right\|^{2}+\operatorname{tr}\left(\Psi_{i}\right)\right) \varphi^{2} I \\
& +2 \sum_{q=1}^{L} \delta_{i q}\left\{\mathbf{Z}_{i r q}^{\mathrm{T}} \Lambda \mathbb{P}_{i} \Lambda \mathbf{Z}_{i r q}+\mathbf{Z}_{i r q}^{\mathrm{T}} \Lambda T^{\mathrm{T}} \mathbb{W}_{i} T \Lambda \mathbf{Z}_{i r q}\right. \\
& \left.+\gamma_{i r} K_{q}^{\mathrm{T}} \Psi_{i} K_{q}+\zeta_{i r} K_{q}^{\mathrm{T}} \Psi_{i} K_{q}\right\}<0 .
\end{aligned}
$$

Clearly, the following inequalities are sufficient conditions for the inequality (38) to hold:

$\mathbf{Z}_{i r q}^{\mathrm{T}} \Lambda \mathbb{P}_{i} \Lambda \mathbf{Z}_{i r q}+\mathbf{Z}_{i r q}^{\mathrm{T}} \Lambda T^{\mathrm{T}} \mathbb{W}_{i} T \Lambda \mathbf{Z}_{i r q}+\gamma_{i r} K_{q}^{\mathrm{T}} \Psi_{i} K_{q}$ 


$$
\begin{aligned}
& +\zeta_{i r} K_{q}^{\mathrm{T}} \Psi_{i} K_{q} \leq Q_{i r q}, \\
& -P_{i}+2 \gamma_{i r}\left(2+2\left\|\Gamma_{i}\right\|^{2}+\operatorname{tr}\left(\Psi_{i}\right)\right) \varphi^{2} I+2 \sum_{q=1}^{L} \delta_{i q} Q_{i r q}<0 .
\end{aligned}
$$

Next, we denote $\bar{P}_{i} \triangleq P_{i}^{-1}, \bar{W}_{i} \triangleq W_{i}^{-1}, \bar{Q}_{i r q} \triangleq Q_{i r q}^{-1}$, $\bar{\gamma}_{i r} \triangleq \gamma_{i r}^{-1}, \bar{\zeta}_{i r} \triangleq \zeta_{i r}^{-1}$, and $\mathcal{K}_{q} \triangleq K_{q} J_{q}$. By resorting to the Schur complement and the following matrix inequality:

$$
\left(J_{q}^{\mathrm{T}}-Q_{\text {irq }}^{-1}\right) Q_{\text {irq }}\left(J_{q}-Q_{\text {irq }}^{-1}\right) \geq 0, i \in \Theta, r \in \mathbb{M}_{n}, q \in \Xi,
$$

it is concluded that the inequalities (34)-(36) hold because of the conditions (29)-(31), and the inequalities (39)-(40) are guaranteed by the conditions (32)-(33), respectively. The proof is now complete.

\section{E. Genetic-algorithm-assisted design algorithm}

Notice that it is difficult to solve the proposed SMC design problem by directly exploiting Theorem 3 due to the nonlinear constraint conditions (11) and (29) as well as the nonconvex coupling terms in the condition (32), e.g., $J_{q}^{\mathrm{T}} \Lambda^{-1} G^{\mathrm{T}} Z_{r}^{-} \Lambda$, $J_{q}^{\mathrm{T}} \Lambda^{-1} G^{\mathrm{T}} Z_{r}^{-} \Lambda T^{\mathrm{T}}$.

Fortunately, the coupled matrix inequalities (30)-(33) reduce to be coupled LMIs if the sliding mode matrix $T \in$ $\mathbb{R}^{m \times n}$ and the matrix $G \in \mathbb{R}^{n \times n}$ are specified a priori, and the corresponding numerical difficulty scales down to the feasible solution to certain coupled LMIs. On the other hand, it is widely known that many existing evolutionary algorithms are able to effectively deal with various optimization problems subject to nonlinear constraints. Some popular evolutionary algorithms include genetic algorithms (GAs) [10], [34] and particle swarm optimization (PSO) algorithms [19]. As such, it is quite natural to combine GAs and LMIs to solve the proposed SMC design problem under the nonlinear constraint conditions (11) and (29).

In order to take advantage of the GAs, an adequate objective function should be given for a certain formulated optimization problem. Notice that the reachability of the sliding region $\mathcal{O}$ in (23) just reflects the SMC performance. Therefore, we consider the following minimization problem for the proposed SMC design:

$$
\min _{T, G} \check{\epsilon}
$$

subject to: LMIs (30)-(33),

$$
\text { and constraints (11), (29), }
$$

where

$$
\begin{aligned}
\check{\epsilon} \triangleq & \max _{i \in \Theta, r \in \mathbb{M}_{n}}\left\{\sqrt{\frac{\bar{\epsilon}_{i r}}{\lambda_{\min }\left(W_{i}\right)}}\right\}, \\
\bar{\epsilon}_{i r} \triangleq & 2\left[\lambda_{\max }\left(B^{\mathrm{T}} \Lambda^{-1} Z_{r} \Lambda \mathbb{P}_{i} \Lambda Z_{r} \Lambda^{-1} B\right)\right. \\
& \left.+\lambda_{\max }\left(B^{\mathrm{T}} \Lambda^{-1} Z_{r} \Lambda T^{\mathrm{T}} \mathbb{W}_{i} T \Lambda Z_{r} \Lambda^{-1} B\right)\right] \\
& \times\left(2+2\left\|\Gamma_{i}\right\|^{2}+\operatorname{tr}\left(\Psi_{i}\right)\right) \varphi^{2} .
\end{aligned}
$$

Remark 7: It is worth mentioning that the nonconvex conditions (11) and (29) are actually difficulty to be solved directly. In the existing literature, the nonconvex condition (11) is always addressed via trial-and-error way or some specific structures, e.g., $L=B^{\mathrm{T}} P$ with Lyapunov matrix $P$ to be determined [23], [32] or $L=B^{\mathrm{T}} X$ with a given matrix $X>0$ [33], [35]. Besides, the nonconvex condition (29) is overcome in the existing literature by trial-and-error way or iterative LMI approach [8], [16]. However, these existing methods may be conservative to the controller design. Compared with the aforementioned results, the present method of introducing GA to the design in this paper can not only reduce the conservative but also produce an optimized sliding mode controller in the sense of the optimization problem (41). This fact actually shows that the proposed GA-assisted SMC design approach is more "smarter" than the ones in the existing literature [23], [32]-[35].

Based upon the above objective function, the binary-based GA is formulated in Algorithm 1 to solve the proposed SMC design problem with coupled LMIs.

Remark 8: In this paper, the SMC problem is investigated for state-saturated systems over the HMFCs. Actually, there are two technical obstacles to solve the specified SMC problem, that is, the state saturation reflecting in the non-convex condition (29) and the HMFCs leading to the asynchronous SMC law (12). The main results proposed in Theorems 13 exhibit the following distinctive merits: 1) the designed SMC law is dependent on the estimated network mode, which is a kind of non-synchronization with the actual network mode via a HMM; 2) the reachability of a sliding domain around the specified sliding surface is analyzed by a stochastic Lyapunov function; 3) the HMFCs and the state saturation nonlinearities are tackled by using the HMM approach and some properties of the Hadamard product; and 4) the proposed SMC problem subject to nonconvex constraint conditions can be solved effectively by the developed GA in combination with the coupled LMIs.

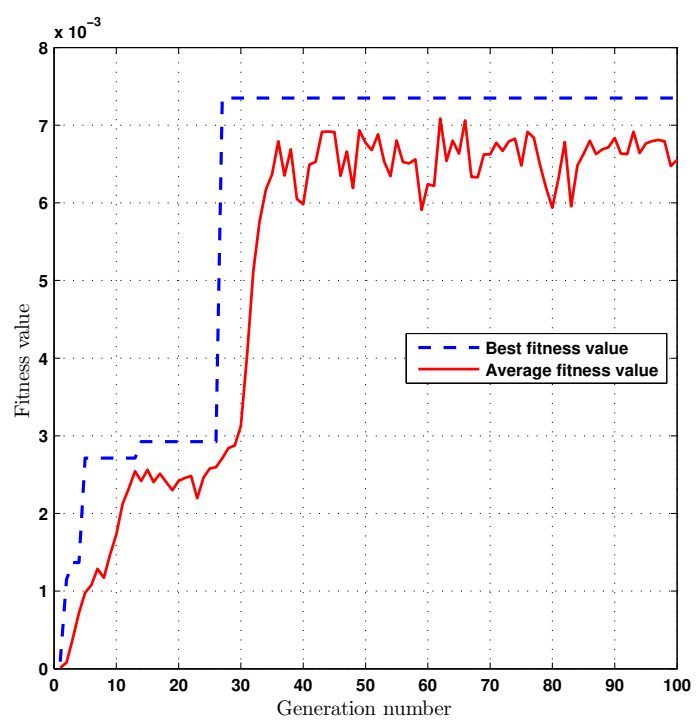

Fig. 3. Fitness value of each generation in solving Algorithm 1. 

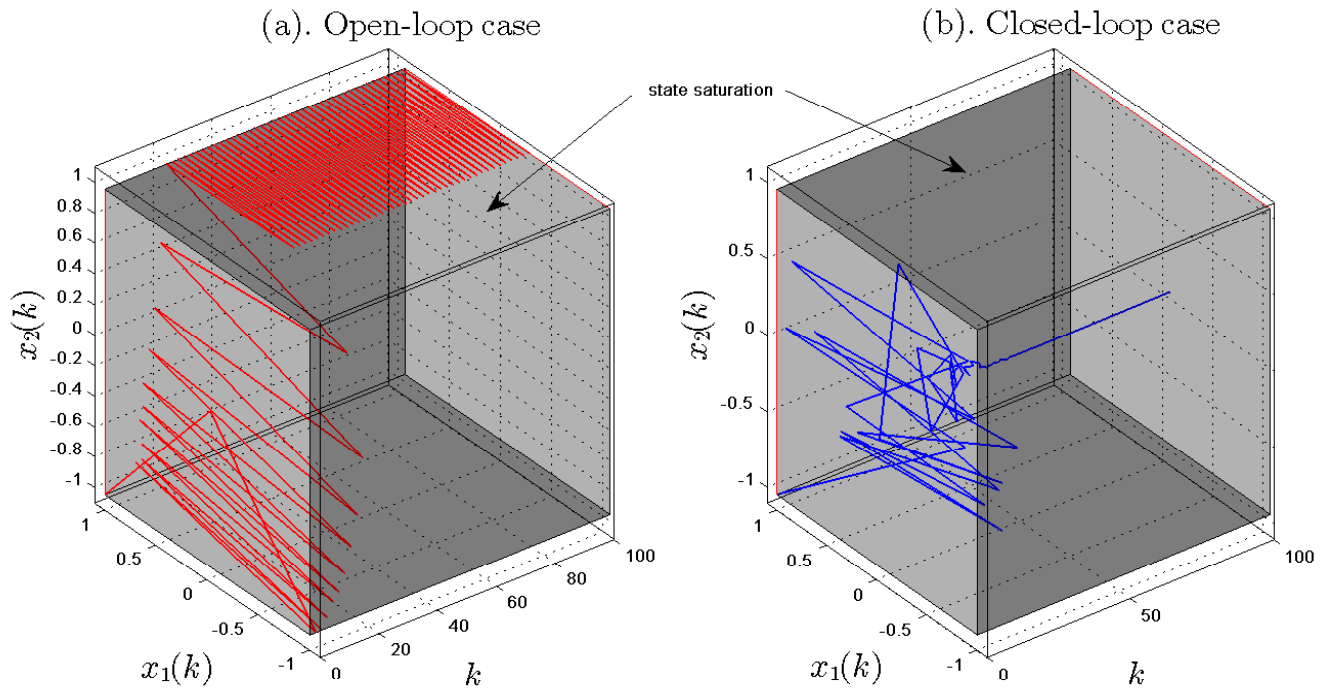

Fig. 4. State trajectories $x(k)$ in open- and closed-loop cases

\section{An NumERICAL EXAMPLE}

Consider a state-saturated system in form of (1) with

$$
\begin{aligned}
& A=\left[\begin{array}{ll}
-1.5 & 1 \\
-0.3 & 1.3
\end{array}\right], B=\left[\begin{array}{l}
1 \\
1
\end{array}\right], \\
& M=\left[\begin{array}{ll}
0.2 & -0.3
\end{array}\right]^{\mathrm{T}}, H=\left[\begin{array}{ll}
-0.1 & 0.09
\end{array}\right], \\
& F(k)=\cos (k), \phi(x(k), k)=0.2 \sqrt{x_{1}^{2}+x_{2}^{2}} .
\end{aligned}
$$

The saturation levels are taken as $\bar{\varpi}_{1}=\bar{\varpi}_{2}=1$ and the bound coefficient of nonlinear function $\phi(x(k), k)$ is set to be $\varphi=0.2$. In this example, we utilize a two-mode ("good" and "bad") Markov fading channel with the transition probability matrix:

$$
\Pi=\left[\begin{array}{ll}
0.6 & 0.4 \\
0.4 & 0.6
\end{array}\right]
$$

When the network channel is in the "good" mode $(\varsigma(k)=1)$, the channel fading variable $\vartheta_{1}(k)$ has the mathematical expectation $\Gamma_{1}=\mu_{1,1}=0.8$ and variance $\Phi_{1}=\Psi_{1}=\xi_{11,1}=0.09$ while, if the network channel is in the "bad" mode $(\varsigma(k)=2)$, the channel fading variable $\vartheta_{2}(k)$ has the mathematical expectation $\Gamma_{2}=\mu_{1,2}=0.2$ and variance $\Phi_{2}=\Psi_{2}=\xi_{11,2}=0.1$.

Now, we utilize a mode detector to estimate the states of the Markov fading channel, where the mode detection probability matrix is given as follows:

$$
\Omega=\left[\begin{array}{ll}
0.5 & 0.5 \\
0.3 & 0.7
\end{array}\right] .
$$

Specifically, when the network channel is in the "good" mode $(\varsigma(k)=1)$, the mode detector has 0.5 probability to emit the "good" mode $(\theta(k)=1)$ or the "bad" mode $(\theta(k)=2)$ to the controller. This is similar to the case that the network channel is in the "bad" mode $(\varsigma(k)=2)$.

The objective of this example is to synthesize an SMC law (12) to stabilize the networked state-saturated system subject to the above HMFC. GA is recalled to solve the proposed SMC design problem. In GA, the parameters are set as follows: population size $N c=50$; maximum of generations $T_{\max }=100$; crossover probability $p_{c}=0.8$; mutation probability $p_{m}=0.1$; bounds of the elements $\bar{t}_{11}=\bar{t}_{12}=5$ and $\underline{t}_{11}=\underline{t}_{12}=-5$; and lengths of binary strings $\ell_{t_{11}}=\ell_{t_{12}}=12$ and $\ell_{g_{11}}=\ell_{g_{12}}=\ell_{g_{21}}=\ell_{g_{22}}=10$. By executing Algorithm 1 , we obtain the following optimized solutions:

$$
\begin{aligned}
& T=\left[\begin{array}{ll}
3.8938 & 4.5092
\end{array}\right], \\
& G=\left[\begin{array}{cc}
-0.3568 & 0.2356 \\
0.6872 & 0.1711
\end{array}\right], \\
& v(k)=\left\{\begin{array}{ll}
{\left[\begin{array}{ll}
1.4847 & -1.5954
\end{array}\right] x(k)} \\
-0.2\|x(k)\| \cdot \operatorname{sgn}(s(k)), \\
{\left[\begin{array}{ll}
1.6680 & -1.8379
\end{array}\right] x(k)} \\
-0.2\|x(k)\| \cdot \operatorname{sgn}(s(k)),
\end{array} \quad \theta(k)=1 ;\right.
\end{aligned}
$$

Fig. 3 depicts the best and average fitness values of each generation in the computation process of GA.

The simulation results for the initial condition $x(0)=$ $\left[\begin{array}{cc}1 & -1\end{array}\right]^{\mathrm{T}}$ are given in Figs. 4-8. It is clear from the Fig. 4(a) that the state-saturated system in open-loop case is unstable. As expected, as shown in Fig. 4(b), the designed SMC law (42) stabilizes the state-saturated system under a possible fading state and detection mode sequence in Fig. 5 and the channel fading variable $\vartheta_{\varsigma(k)}(k)$ in Fig. 6. The reachability of the sliding region $\mathcal{O}$ in (23) is clearly illustrated in Fig. 7. Fig. 8 shows the SMC signal $v(k)$ and the actual control signal $u(k)$ simultaneously. To this end, the effectiveness of the proposed GA-based state-saturated SMC scheme subject to the HMFCs is validated from all obtained simulation results.

\section{Conclusions}

This paper has dealt with the SMC problem for networked state-saturated systems over the finite-state HMFCs. The mode detector has been employed to estimate the actual network mode via a HMM. Based on the estimated network modes, a suitable SMC scheme has been proposed with a linear sliding 

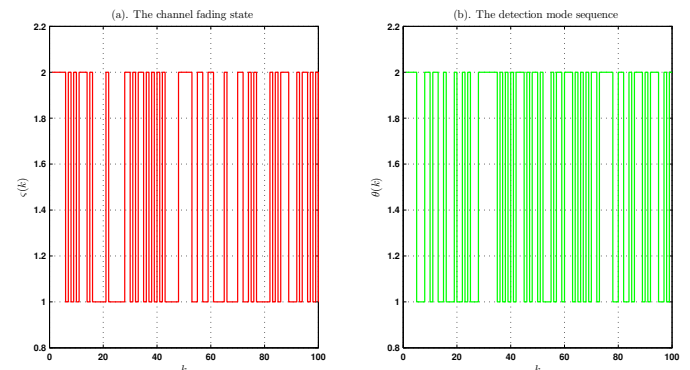

Fig. 5. A possible fading state and detection mode sequence

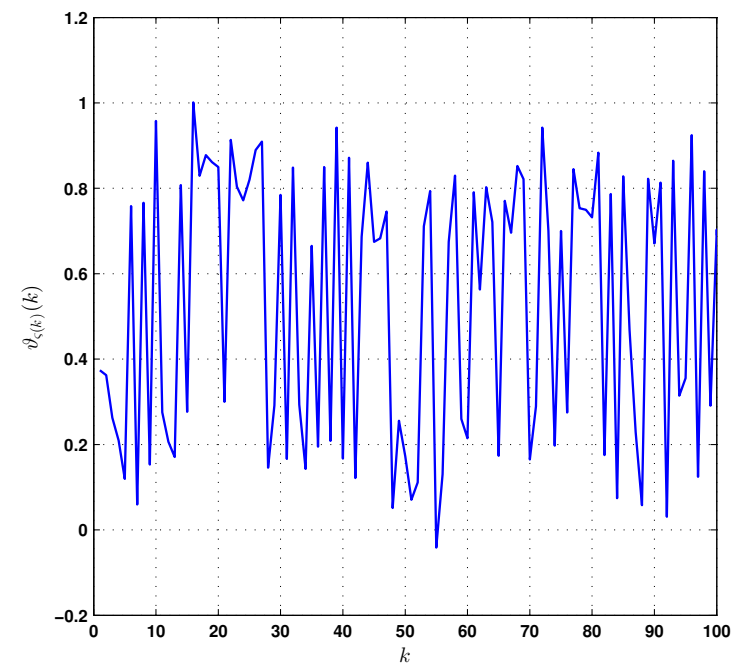

Fig. 6. The channel fading variable $\vartheta_{\varsigma(k)}(k)$

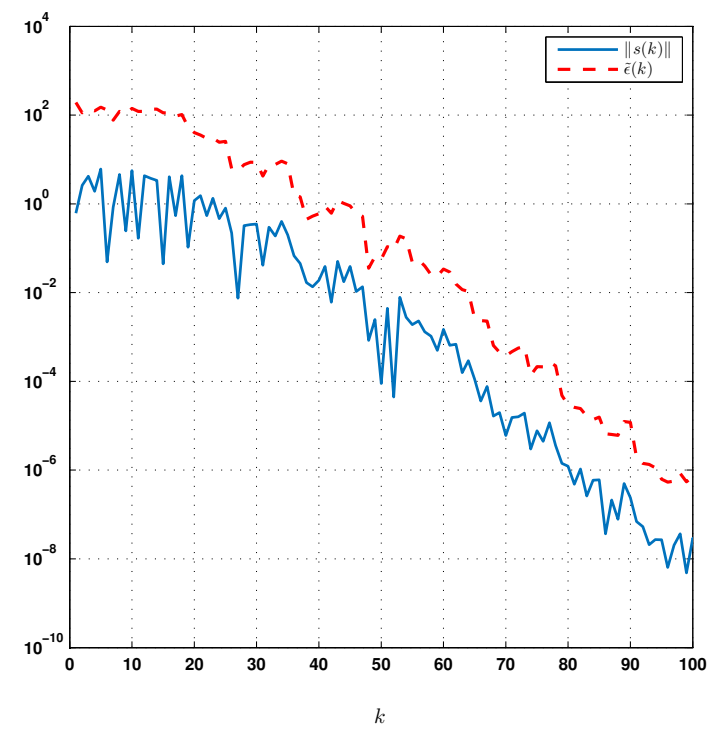

Fig. 7. Illustration of the reachability to the sliding region $\mathcal{O}$ in (23) \begin{tabular}{l}
\hline Algorithm 1 GA-Assisted State-Saturated SMC Design \\
\hline Step 1: Parameter encoding. Denote the sliding gain \\
matrix $T=\left[t_{i j}\right]_{m \times n}$ and the matrix $G=\left[g_{i j}\right]_{n \times n}$, so \\
there are $m n+n^{2}$ independent variables. To this end, the \\
phenotype in the search space is expressed as a row vector \\
$w \in \mathbb{R}^{1 \times\left(m n+n^{2}\right)}$ :
\end{tabular}

$$
\begin{aligned}
& {[T, G] \rightarrow w \triangleq\left[\begin{array}{llllll}
t_{11} & \ldots & t_{1 n} & t_{21} & \ldots & t_{m n}
\end{array}\right.} \\
& \left.\begin{array}{llllll}
g_{11} & \ldots & g_{1 n} & g_{21} & \ldots & g_{n n}
\end{array}\right] \text {. }
\end{aligned}
$$

In $w$, each element $t_{i j}$ is coded as a binary string with the length $\ell_{t_{i j}}$ over the range of $t_{i j} \in\left[\underline{t}_{i j}, \bar{t}_{i j}\right]$. The precision $q_{t_{i j}}$ under the linearly-mapped coding can be obtained by $q_{t_{i j}}=\frac{\bar{t}_{i j}-\underline{t}_{i j}}{2^{\ell_{i j}}-1}$. Furthermore, it is noted that the nonlinear constraint condition (29) implies that $g_{i j} \in[-1,1]$. Hence, for each element $g_{i j}$ in $w$ is coded as a binary string of length $\ell_{g_{i j}}$, the precision $q_{g_{i j}}$ can be computed as $q_{g_{i j}}=$ $\frac{2}{2^{\ell g_{i j}}-1}$.

Step 2: Population initialization. Initial population of $N c$ chromosomes $w_{l}, l=1,2, \ldots, N c$, is generated randomly.

- Step 3: Fitness function and assignment. Decode the initial population produced in Step 2 into a real values for every phenotype, and then the fitness function $\operatorname{Fitness}\left(T_{l}, G_{l}\right) \triangleq \frac{1}{\tilde{\epsilon}}$ is computed for every $T_{l}$ and $G_{l}$ via solving coupled LMIs (30)-(33). If either the coupled LMIs (30)-(33) are infeasible or the constraint conditions (11), (29) are not hold, then the fitness function Fitness $\left(T_{l}, G_{l}\right)$ will be artificially assigned a sufficiently small value $\left(10^{-6}\right.$ in this paper) for reducing its opportunity to survive in the next generation.

- Step 4: Performing genetic operations. According to the assigned fitness in Step 3, we obtain the next population by executing the sequence of genetic operations Selection, Crossover and Mutation, respectively (more details can be found in [10], [34]). Here, we denote the single-point crossover probability as $p_{c}$, and a single bit mutation probability as $p_{m}$.

- Step 5: Stop criterion. The evolution process will be repeated from Step 3 to 4 in each generation until the maximum generations $N_{\max }$ is reached. And then, decode the best chromosome $w_{l}$ into real values with producing the sliding gain matrix $T$ and the matrix $G$.

- Step 6: Design of SMC laws. Produce the SMC law (12) by using the sliding gain matrix $T$ and the gain matrices $K_{q}=\mathcal{K}_{q} J_{q}^{-1}$ obtained from Step 5, and then apply it to stabilize the state-saturated system (1) subject to the HMFCs (5)-(9).

surface. By resorting to the stochastic Lyapunov function and the HMM approach, the sufficient conditions have been derived to ensure both the mean-square stability of the sliding mode dynamics and the reachability of the specified sliding surface. Furthermore, with the aid of the polytopic representation of the saturation nonlinearities and some properties of the Hadamard product, a GA combining with LMIs has been proposed to solve the SMC design problem subject to some 


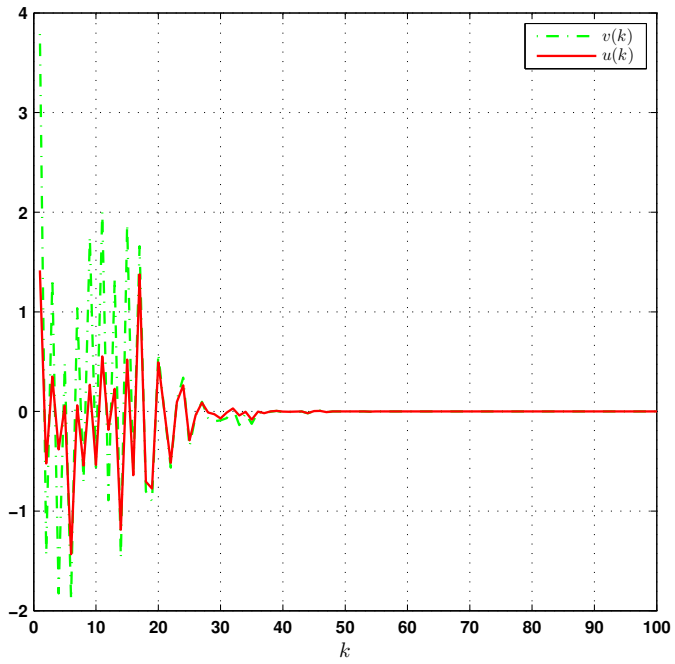

Fig. 8. The SMC input $v(k)$ and the actual control input $u(k)$

nonconvex constraint conditions. In the near future, we may extend the proposed results to the state-saturated SMC issues under the fading measurements [6], [26] and employ some improved evolutionary algorithms [19] to solve the proposed non-convex optimization problem more effectively.

\section{REFERENCES}

[1] M. V. Basin and P. C. Rodríguez-Ramírez, Sliding mode controller design for stochastic polynomial systems with unmeasured states, IEEE Transactions on Industrial Electronics, vol. 61, no. 1, pp. 387-396, 2014.

[2] Z. Cao, Y. Niu and J. Song, Finite-time sliding mode control of Markovian jump cyber-physical systems against randomly occurring injection attacks, IEEE Transactions on Automatic Control, vol. 65, no. 3, pp. 1264-1271, 2020.

[3] Z. Cao, Y. Niu and H. Zhao. Finite-time sliding mode control of Markovian jump systems subject to actuator faults. International Journal of Control, Automation and Systems, vol. 16, no. 5, pp. 2282-2289, 2018.

[4] Y. Chen, Z. Wang, Y. Yuan and P. Date, Distributed $H_{\infty}$ filtering for switched stochastic delayed systems over sensor networks with fading measurements, IEEE Transactions on Cybernetics, vol. 50, no. 1, pp. 214, Jan. 2020.

[5] O. L. V. Costa, M. D. Fragoso and M. G. Todorov, A detector-based approach for the $\mathrm{H}_{2}$ control of Markov jump linear systems with partial information, IEEE Transactions on Automatic Control, vol. 60, no. 5, pp. 1219-1234, 2015.

[6] M. Dawa, G. Kaddoum and Z. Sattar, A generalized lower bound on the bit error rate of DCSK systems over multi-path Rayleigh fading channels, IEEE Transactions on Circuits and Systems II: Express Briefs, vol. 65, no. 3, pp. 321-325, 2018.

[7] A. M. de. Oliveira and O. L. V. Costa, $\mathrm{H}_{2}$-filtering for discrete-time hidden Markov jump systems, International Journal of Control, vol. 90, no. 3, pp. 599-615, 2017.

[8] D. Ding, Z. Wang, B. Shen and H. Shu, State-saturated $H_{\infty}$ filtering with randomly occurring nonlinearities and packet dropouts: the finitehorizon case, International Journal of Robust and Nonlinear Control, vol. 23, no. 16, pp. 1803-1821, 2013.

[9] D. Ding, Z. Wang, J. Lam and B. Shen, Finite-horizon $H_{\infty}$ control for discrete time-varying systems with randomly occurring nonlinearities and fading measurements, IEEE Transactions on Automatic Control, vol. 60 , no. 9, pp. 2488-2493, 2015.

[10] H. Du, J. Lam and K. Y. Sze, Non-fragile output feedback $H_{\infty}$ vehicle suspension control using genetic algorithm, Engineering Applications of Artificial Intelligence, vol. 16, no. 7-8, pp. 667-680, 2003.

[11] N. Elia, Remote stabilization over fading channels, Systems \& Control Letter, vol. 54, no. 3, pp. 237-249, 2005.
[12] R. A. Horn and C. R. Johnson, Matrix Analysis (Second Edition), Cambridge University Press, Cambridge, 2013.

[13] J. Hu, Z. Wang, H. Gao and L. K. Stergioulas, Robust sliding mode control for discrete stochastic systems with mixed time delays, randomly occurring uncertainties, and randomly occurring nonlinearities, IEEE Transactions on Industrial Electronics, vol. 59, no. 7, pp. 3008-3015, 2012.

[14] P. Ignaciuk and A. Bartoszewicz, Discrete-time sliding-mode congestion control in multisource communication networks with time-varying delay, IEEE Transactions on Control Systems Technology, vol. 19, no. 4, pp. 852-867, 2011.

[15] G. P. Incremona, M. Cucuzzella and A. Ferrara, Second order sliding mode control for nonlinear affine systems with quantized uncertainty, Automatica, vol. 86, pp. 46-52, 2017.

[16] X. Ji, T. Liu, Y. Sun and H. Su, Stability analysis and controller synthesis for discrete linear time-delay systems with state saturation nonlinearities, International Journal of Systems Science, vol. 42, no. 3, pp. 397-406, 2011.

[17] B. Jiang, H. R. Karimi, Y. Kao and C. Gao, A novel robust fuzzy integral sliding mode control for nonlinear semi-Markovian jump TS fuzzy systems, IEEE Transactions on Cybernetics, vol. 26, no. 6, pp. 3594-3604, 2018.

[18] S. Liu, Z. Wang, G. Wei and M. Li, Distributed set-membership filtering for multi-rate systems under the Round-Robin scheduling over sensor networks, IEEE Transactions on Cybernetics, in press, DOI: $10.1109 /$ TCYB.2018.2885653.

[19] W. Liu, Z. Wang, Y. Yuan, N. Zeng, K. Hone and X. Liu. A novel sigmoid-function-based adaptive weighted particle swarm optimizer, IEEE Transactions on Cybernetics, 2019, DOI: 10.1109/TCYB.2019.2925015.

[20] X. Liu, X. Yu, G. Ma and H. Xi, On sliding mode control for networked control systems with semi-Markovian switching and random sensor delays, Information Sciences, vol. 337-338, pp. 44-58, 2016.

[21] M. Liu, L. Zhang, P. Shi and Y. Zhao, Sliding mode control of continuous-time Markovian jump systems with digital data transmission, Automatica, vol. 80, pp. 200-209, 2017.

[22] Z. Ning, L. Zhang, J. de. J. Rubio and X. Yin, Asynchronous filtering for discrete-time fuzzy affine systems with variable quantization density, IEEE Transactions on Cybernetics, vol. 47, no. 1, pp. 153-164, 2017.

[23] Y. Niu and D. W. C. Ho, Design of sliding mode control subject to packet losses, IEEE Transactions on Automatic Control, vol. 55, no. 11, pp. 2623-2628, 2010.

[24] M. Ogura, A. Cetinkaya, T. Hayakawa and V. M. Preciado, Statefeedback control of Markov jump linear systems with hidden Markov mode observation, Automatica, vol. 89, pp. 65-72, 2018.

[25] J. Qiu, Y. Wei, H. R. Karimi and H. Gao, Reliable control of discretetime piecewise-affine time-delay systems via output feedback, IEEE Transactions on Reliability, vol. 67, no. 1, pp. 79-91, 2018.

[26] D. E. Quevedo, A. Ahlén and K. H. Johansson, State estimation over sensor networks with correlated wireless fading channels, IEEE Transactions on Automatic Control, vol. 58, no. 3, pp. 581-593, 2013.

[27] P. Sadeghi, R. A. Kennedy, P. B. Rapajic and R. Shams, Finite-state Markov modeling of fading channels: A survey of principles and applications, IEEE Signal Processing Magazine, vol. 25, no. 5, pp. $57-$ 80, 2008.

[28] B. Shen, Z. Wang, D. Wang and Q. Li. State-saturated recursive filter design for stochastic time-varying nonlinear complex networks under deception attacks. IEEE Transactions on Neural Networks and Learning Systems, 2019, DOI: 10.1109/TNNLS.2019.2946290.

[29] B. Shen, Z. Wang, D. Wang and H. Liu. Distributed state-saturated recursive filtering over sensor networks under Round-Robin protocol. IEEE Transactions on Cybernetics, 2019, DOI: 10.1109/TCYB.2019.2932460.

[30] B. Shen, Z. Wang, D. Wang, J. Luo, H. Pu and Y. Peng. Finite-horizon filtering for a class of nonlinear time-delayed systems with an energy harvesting sensor, Automatica, vol. 100, no. 2, pp. 144-152, 2019.

[31] Y. Shen, Z. Wang, B. Shen, F. E. Alsaadi and F. E. Alsaadi, Fusion estimation for multi-rate linear repetitive processes under weighted Try-Once-Discard protocol, Information Fusion, vol. 55, pp. 281-291, Mar. 2020.

[32] H. Song, S. C. Chen and Y. Yam, Sliding mode control for discretetime systems with Markovian packet dropouts, IEEE Transactions on Cybernetics, vol. 47, no. 11, pp. 3669-3679, 2017.

[33] J. Song and Y. Niu. Dynamic event-triggered sliding mode control: Dealing with slow sampling singularly perturbed systems. IEEE Transactions on Circuits and Systems II: Express Briefs, 2019, DOI: 10.1109/TCSII.2019.2926879. 
[34] J. Song and Y. Niu. Co-design of 2D event generator and sliding mode controller for 2D Roesser model via genetic algorithm. IEEE Transactions on Cybernetics, 2020, DOI: 10.1109/TCYB.2019.2959139.

[35] J. Song, Y. Niu and H.-K. Lam. Reliable sliding mode control of fast sampling singularly perturbed systems: A redundant channel transmission protocol approach. IEEE Transactions on Circuits and Systems I: Regular Papers, vol. 66, no. 11, pp. 4490-4501, 2019.

[36] J. Song, Y. Niu and Y. Zou, A parameter-dependent sliding mode approach for finite-time bounded control of uncertain stochastic systems with randomly varying actuator faults and its application to a parallel active suspension system, IEEE Transactions on Industrial Electronics, vol. 65, no. 10, pp. 8124-8132, 2018.

[37] J. Song, Y. Niu and J. Xu. An event-triggered approach to sliding mode control of Markovian jump Lur'e systems under hidden mode detections. IEEE Transactions on Systems, Man, and Cybernetics: Systems, 2018, DOI: $10.1109 /$ TSMC.2018.2847315.

[38] N. Xiao, Y. Niu and L. Xie, State feedback stabilization over finite-state fading channels, Asian Journal of Control, vol. 18, no. 3, pp. 1052-1061, 2016.

[39] N. Xiao, L. Xie and L. Qiu, Feedback stabilization of discrete-time networked systems over fading channels, IEEE Transactions on Automatic Control, vol. 57, no. 9, pp. 2176-2189, 2012.

[40] L. Xu, L. Xie and N. Xiao, Mean square stabilization over Gaussian finite-state Markov channels, IEEE Transactions on Control of Network Systems, vol. 5, no. 4, pp. 1830-1840, 2018.

[41] H. Yan, Y. Tian, H. Li, H. Zhang and Z. Li. Input-output finite-time mean square stabilisation of nonlinear semi-Markovian jump systems with time-varying delay. Automatica, vol. 104, pp. 82-89, 2019.

[42] F. Yang, Q.-L. Han and Y. Liu. Distributed $H_{\infty}$ state estimation over a filtering network with time-varying and switching topology and partial information exchange. IEEE Transactions on Cybernetics, vol. 49, no. 3, pp. 870-882, 2019.

[43] Y. Yuan, P. Zhang, Z. Wang and L. Guo, On resilient strategy design of multi-tasking optimal control for state-saturated systems with nonlinear disturbances: the time-varying case, Automatica, vol. 107, pp. 138-145, Sept. 2019.

[44] X. M. Zhang, Q. L. Han and X. Yu, Survey on recent advances in networked control systems, IEEE Transactions on Industrial Informatics, vol. 12, no. 5, pp. 1740-1752, 2016.

[45] Z. Zhang and Y. Niu. Adaptive sliding mode control for interval type2 stochastic fuzzy systems subject to actuator failures. International Journal of Systems Science, vol. 49, no. 15, pp. 3169-3181, 2018.

[46] H. Zhang, Z. Wang, H. Yan, F. Yang and X. Zhou. Adaptive eventtriggered transmission scheme and $H_{\infty}$ filtering co-design over a filtering network with switching topology. IEEE Transactions on Cybernetics, vol. 49, no. 12, pp. 4296-4307, 2019.

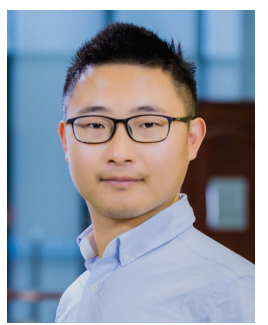

Jun Song (M'19) received the B.E. degree in electronic science and technology and the M.E. degree in pattern recognition and intelligent system from Anhui University, Hefei, China, in 2011 and 2014, respectively, and the Ph.D. degree in control science and engineering from East China University of Science and Technology, Shanghai, China, in 2018.

$\mathrm{He}$ is currently an Associate Professor with East China University of Science and Technology. From October 2016 to October 2017, he was a visiting Ph.D. student with the Department of Computer Science, Brunel University London, Uxbridge, U.K. He also was a Research Assistant or Research Fellow with The University of Hong Kong, Hong Kong, City University of Hong Kong, Hong Kong, and Western Sydney University, Penrith, NSW, Australia. His research interests including networked sliding mode control theory and its applications, Markovian jump systems, large-scale systems, and finite-time stability. He is a member of CAA and IEEE.

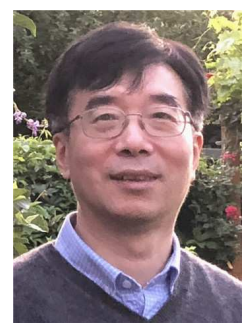

Zidong Wang (SM'03-F'14) was born in Jiangsu, China, in 1966. He received the B.Sc. degree in mathematics in 1986 from Suzhou University, Suzhou, China, and the M.Sc. degree in applied mathematics in 1990 and the Ph.D. degree in electrical engineering in 1994, both from Nanjing University of Science and Technology, Nanjing, China.

$\mathrm{He}$ is currently Professor of Dynamical Systems and Computing in the Department of Information Systems and Computing, Brunel University London, U.K. From 1990 to 2002, he held teaching and research appointments in universities in China, Germany and the UK. Prof. Wang's research interests include dynamical systems, signal processing, bioinformatics, control theory and applications. He has published more than 500 papers in refereed international journals. He is a holder of the Alexander von Humboldt Research Fellowship of Germany, the JSPS Research Fellowship of Japan, William Mong Visiting Research Fellowship of Hong Kong.

Prof. Wang serves (or has served) as the Editor-in-Chief for Neurocomputing, the Deputy Editor-in-Chief for International Journal of Systems Science, and an Associate Editor for 12 international journals, including IEEE Transactions on Automatic Control, IEEE Transactions on Control Systems Technology, IEEE Transactions on Neural Networks, IEEE Transactions on Signal Processing, and IEEE Transactions on Systems, Man, and Cybernetics - Part C. He is a Fellow of the IEEE, a Fellow of the Royal Statistical Society and a member of program committee for many international conferences.

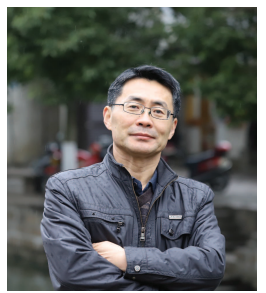

Yugang Niu received his M.Sc. and Ph.D. degrees in control engineering from the Nanjing University of Science and Technology, Nanjing, China, in 1992 and 2001, respectively.

In 2003, he joined the School of Information Science and Engineering, East China University of Science and Technology, Shanghai, China, where he is currently a Professor. His current research interests include stochastic systems, sliding mode control, Markovian jump systems, networked control systems, wireless sensor networks, and smart grid.

Prof. Niu is currently an Associate Editor of several international journals, including Information Sciences, Neurocomputing, IET Control Theory \& Applications, Journal of The Franklin Institute, and International Journal of System Sciences. He is also a member of Conference Editorial Board of IEEE Control Systems Society.

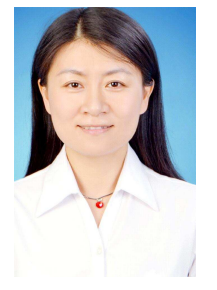

Hongli Dong (SM'16) received the Ph.D. degree in control science and engineering from the Harbin Institute of Technology, Harbin, China, in 2012.

From 2009 to 2010, she was a Research Assistant with the Department of Applied Mathematics, City University of Hong Kong, Hong Kong. From 2010 to 2011, she was a Research Assistant with the Department of Mechanical Engineering, The University of Hong Kong, Hong Kong. From 2011 to 2012, she was a Visiting Scholar with the Department of Information Systems and Computing, Brunel University London, London, U.K. From 2012 to 2014, she was an Alexander von Humboldt Research Fellow with the University of Duisburg-Essen, Duisburg, Germany. She is currently a Professor with the Institute of Complex Systems and Advanced Control, Northeast Petroleum University, Daqing, China. She is also the Director of the Heilongjiang Provincial Key Laboratory of Networking and Intelligent Control, Daqing. Her current research interests include robust control and networked control systems.

Dr. Dong is a very active reviewer for many international journals. 